\title{
Photoreceptive Ganglion Cells Drive Circuits for Local Inhibition in the Mouse Retina
}

\author{
${ }^{\circledR}$ Joseph Pottackal, ${ }^{1}$ Hannah L. Walsh, ${ }^{2}$ Pouyan Rahmani, ${ }^{2}{ }^{\circledR}$ Kathy Zhang, ${ }^{1}$ Nicholas J. Justice, ${ }^{5}$ and \\ (ㄱ) Jonathan B. Demb $b^{1,2,3,4}$ \\ ${ }^{1}$ Interdepartmental Neuroscience Program, ${ }^{2}$ Department of Ophthalmology and Visual Science, ${ }^{3}$ Department of Cellular and Molecular Physiology, \\ ${ }^{4}$ Department of Neuroscience, Yale University, New Haven, Connecticut 06511, and ${ }^{5}$ Institute of Molecular Medicine, University of Texas Health \\ Science Center, Houston, Texas 77030
}

Intrinsically photosensitive retinal ganglion cells (ipRGCs) exhibit melanopsin-dependent light responses that persist in the absence of rod and cone photoreceptor-mediated input. In addition to signaling anterogradely to the brain, ipRGCs signal retrogradely to intraretinal circuitry via gap junction-mediated electrical synapses with amacrine cells (ACs). However, the targets and functions of these intraretinal signals remain largely unknown. Here, in mice of both sexes, we identify circuitry that enables M5 ipRGCs to locally inhibit retinal neurons via electrical synapses with a nonspiking GABAergic AC. During pharmacological blockade of rod- and cone-mediated input, whole-cell recordings of corticotropin-releasing hormone-expressing $\left(\mathrm{CRH}^{+}\right) \mathrm{ACs}$ reveal persistent visual responses that require both melanopsin expression and gap junctions. In the developing retina, ipRGC-mediated input to $\mathrm{CRH}^{+} \mathrm{ACs}$ is weak or absent before eye opening, indicating a primary role for this input in the mature retina (i.e., in parallel with rod- and cone-mediated input). Among several ipRGC types, only M5 ipRGCs exhibit consistent anatomical and physiological coupling to $\mathrm{CRH}^{+}$ACs. Optogenetic stimulation of local $\mathrm{CRH}^{+}$ACs directly drives IPSCs in M4 and M5, but not M1-M3, ipRGCs. CRH ${ }^{+}$ACs also inhibit M2 ipRGC-coupled spiking ACs, demonstrating direct interaction between discrete networks of ipRGC-coupled interneurons. Together, these results demonstrate a functional role for electrical synapses in translating ipRGC activity into feedforward and feedback inhibition of local retinal circuits.

Key words: amacrine cell; corticotropin releasing hormone; gap junction; ipRGC; melanopsin; retinal ganglion cell

Significance Statement

Melanopsin directly generates light responses in intrinsically photosensitive retinal ganglion cells (ipRGCs). Through gap junction-mediated electrical synapses with retinal interneurons, these uniquely photoreceptive RGCs may also influence the activity and output of neuronal circuits within the retina. Here, we identified and studied an electrical synaptic circuit that, in principle, could couple ipRGC activity to the chemical output of an identified retinal interneuron. Specifically, we found that M5 ipRGCs form electrical synapses with corticotropin-releasing hormone-expressing amacrine cells, which locally release GABA to inhibit specific RGC types. Thus, ipRGCs are poised to influence the output of diverse retinal circuits via electrical synapses with interneurons.

Received Mar. 23, 2020; revised Nov. 11, 2020; accepted Dec. 18, 2020.

Author contributions: J.P., N.J.J., and J.B.D. designed research; J.P., H.L.W., P.R., and K.Z. performed research; J.P. and H.L.W. analyzed data; J.P. wrote the first draft of the paper; J.P. and J.B.D. edited the paper; J.P. and J.B.D. wrote the paper.

This work was supported by National Institutes of Health Grants EY014454 and EY021372 to J.B.D., P30 EY026878, T32 NS041228, and T32 EY022312; National Science Foundation Graduate Research Fellowship to J.P.; and a Gruber Science Fellowship to J.P. We thank Joshua Singer and Franklin Caval-Holme for helpful comments on the manuscript.

The authors declare no competing financial interests.

Correspondence should be addressed to Jonathan B. Demb at jonathan.demb@yale.edu.

https://doi.org/10.1523/JNEUROSCI.0674-20.2020

Copyright $\odot 2021$ the authors

\section{Introduction}

In mice, intrinsically photosensitive retinal ganglion cells (ipRGCs) comprise a physiologically and morphologically diverse set of up to six cell types (M1-M6) (Ecker et al., 2010; Schmidt et al., 2011; Zhao et al., 2014; Quattrochi et al., 2019) that express melanopsin, which renders them photosensitive in the absence of rod- and cone-mediated input (Berson et al., 2002; Hattar et al., 2002). ipRGC axons project to several brain regions, providing input to both image-forming visual circuits, mediating perception; and non-image-forming visual circuits, mediating light-dependent functions, such as circadian photoentrainment and the pupillary light reflex (Hattar et al., 2006; Güler et al., 2008; Ecker et al., 2010; Chen et al., 2011; Estevez et al., 
2012; LeGates et al., 2012; Stabio et al., 2018; Fernandez et al., 2018; Do, 2019).

Additionally, ipRGCs signal retrogradely to intraretinal circuitry via at least two anatomically distinct pathways. First, M1 ipRGCs make chemical synapses onto dopaminergic amacrine cells (ACs), likely via axon collaterals that project "backwards" into the inner plexiform layer (IPL) (Viney et al., 2007; Zhang et al., 2008; Joo et al., 2013; Prigge et al., 2016). Second, most ipRGC types make electrical synapses, via gap junctions, with multiple AC types whose identities remain mostly unknown (Schubert et al., 2005; Völgyi et al., 2005; Pérez de Sevilla Müller et al., 2010; Reifler et al., 2015; Liao et al., 2016; Sabbah et al., 2017). These ACs are predominantly GABAergic (Pérez de Sevilla Müller et al., 2010; Reifler et al., 2015), and many exhibit wide-field morphology and action potential firing (Pérez de Sevilla Müller et al., 2010; Reifler et al., 2015; Sabbah et al., 2017); thus, it has been postulated that these ACs provide circuitry by which ipRGCs can broadcast their activity globally throughout the retina to enact irradiance-dependent changes in circuit operation (Allen et al., 2014; Tikidji-Hamburyan et al., 2015; Milosavljevic et al., 2018). Evaluation of hypotheses regarding functions of ipRGC-AC coupling, however, has been precluded by poor understanding of the circuit architectures that integrate identified ipRGC-coupled ACs into specific retinal circuits. In principle, identifying retinal circuits that receive input from an ipRGC-coupled AC could clarify the role of the coupled AC in the computations performed by these circuits.

Here, we leverage mouse genetics to uncover three distinct types of inhibitory circuits driven by electrical coupling between the M5 ipRGC and the corticotropin-releasing hormoneexpressing $\left(\mathrm{CRH}^{+}\right) \mathrm{AC}$, a medium-field interneuron whose nonspiking physiology and axonless morphology distinguish it from ipRGC-coupled ACs described previously. This electrical synapse develops near eye opening, concurrently with the expansion of the $\mathrm{CRH}^{+} \mathrm{AC}$ neuritic arbor and with the functional maturation of rod- and cone-driven circuits. $\mathrm{CRH}^{+}$ACs make GABAergic synapses onto M5 ipRGCs, forming a negative feedback mechanism, and also provide feedforward inhibition to M4 ipRGCs (Park et al., 2018) and suppressed-by-contrast RGCs (Jacoby et al., 2015). $\mathrm{CRH}^{+}$ACs also appear to inhibit M2 ipRGC-coupled wide-field ACs (WACs), suggesting that local M5 ipRGC activity could indirectly influence long-range transmission of M2 ipRGC activity within the retina. These results show that, through electrical synapses with $\mathrm{CRH}^{+}$ACs, ipRGC activity locally influences the output of specific RGC circuits in the mature retina.

\section{Materials and Methods}

Animals. All animal procedures were approved by the Institutional Animal Care and Use Committee at Yale University and were in compliance with National Institutes of Health guidelines. Mice of both sexes were maintained on a C57BL/6 background and studied between postnatal days 28 and 90 (P28-P90), except in developmental experiments, for which animals were studied between P9 and P16.

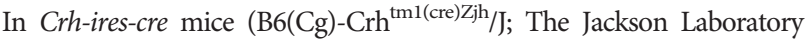
\#012704), endogenous Crh regulatory elements drive expression of Cre recombinase (Taniguchi et al., 2011). Ai14 mice (B6.Cg-Gt (ROSA)26Sor ${ }^{\text {tm14(CAG-tdTomato)Hze } / J ; ~ T h e ~ J a c k s o n ~ L a b o r a t o r y ~}$ \#007914) enabled Cre-dependent expression of the red fluorescent protein tdTomato (tdT) (Madisen et al., 2010). Ai32 mice (B6.Cg-

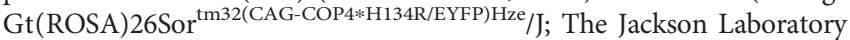
\#024109) enabled Cre-dependent expression of a channelrhodopsin-2 (ChR2)-enhanced yellow fluorescent protein (EYFP) fusion protein (Madisen et al., 2012). In Crhrl-gfp mice, Crhrl regulatory elements genomically introduced via bacterial artificial chromosome drive expression of GFP (Justice et al., 2008). In Opn4-gfp mice ( $\mathrm{Tg}(\mathrm{Opn} 4-$ EGFP)ND100Gsat/Mmucd), Opn4 regulatory elements genomically introduced via bacterial artificial chromosome drive expression of GFP. In Opn4-cre mice, endogenous regulatory elements drive expression of Cre recombinase in place of melanopsin (Ecker et al., 2010); that is, mice homozygous for this allele (Opn $4^{\text {Cre/Cre }}$ ) lack melanopsin expression.

Experimental mouse strains were generated by crossing various combinations of the transgenic mice described above. In $\mathrm{Crh}^{-\mathrm{Cr}^{+/-}} ; \mathrm{Ail4}^{+/-}$ mice, $\mathrm{CRH}^{+}$ACs express tdT (see Figs. 1, 3, 7, 9). In Crh-cre ${ }^{+/-} ; \mathrm{Ai32}^{+/-}$ mice, $\mathrm{CRH}^{+}$ACs express ChR2-EYFP (see Figs. 6, 7, 10, 11). In Opn4-gfp ${ }^{+}$; $\mathrm{Crh}_{-\mathrm{Cre}}^{+/-} ; \mathrm{Ai32}^{+/-}$and Crhr1-gfp ${ }^{+} ; \mathrm{Crh}_{-} \mathrm{cre}^{+/-} ; \mathrm{Ai32} 2^{+/-}$mice, M1-M3 ipRGCs or M5 ipRGCs, respectively, strongly express GFP and $\mathrm{CRH}^{+} \mathrm{ACs}$ express ChR2-EYFP (see Figs. 6-8, 10). In Crhr1-gfp ${ }^{+}{\text {Crh }-\mathrm{cre}^{+/-}}^{+} \mathrm{Ail4}^{+/-}$ mice, $\mathrm{CRH}^{+} \mathrm{ACs}$ and M5 ipRGCs strongly express GFP and $\mathrm{CRH}^{+} \mathrm{ACs}$ additionally express tdT (see Figs. 2, 7). In Crhrl-gfp ${ }^{+} ; \mathrm{Op} n 4^{\mathrm{Cre} / \mathrm{Cre}}$ mice, a subset of $\mathrm{CRH}^{+}$ACs strongly express GFP and melanopsin expression is

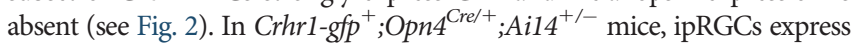
tdT, enabling visualization of overlap between $\mathrm{GFP}^{+}$cells and ipRGCs (see Figs. 4, 5).

Electrophysiology. Before retinal dissection, mice were dark-adapted for $\sim 1 \mathrm{~h}$. Following death, both eyes were enucleated and transferred to a dissection dish filled with Ames medium (A1420, MilliporeSigma), supplemented with $22.6 \mathrm{~mm} \mathrm{NaHCO}_{3}$ (MilliporeSigma) and suffused with a $95 \% \mathrm{O}_{2} / 5 \% \mathrm{CO}_{2}$ gas mixture. Dissections were performed under infrared illumination using stereomicroscope-mounted night-vision goggles. After extracting the retina from the eyecup, the vitreous humor was removed and a single relaxing cut was made along the nasotemporal axis toward the optic disk. In some cases, retinas were cut into dorsal and ventral hemi-retinas. Whole retinas or hemi-retinas were mounted onto mixed cellulose ester filter membranes (HAWP01300, MilliporeSigma) and maintained in the dissection dish at room temperature for up to $5 \mathrm{~h}$. Immediately before recording, retinas were placed into a custom recording chamber and secured by a tissue harp. During experiments, the recording chamber was perfused with Ames medium flowing at 4- $6 \mathrm{ml} / \mathrm{min}$ and maintained at $32^{\circ} \mathrm{C}-34^{\circ} \mathrm{C}$.

Whole-cell patch-clamp recordings from 170 cells were obtained using patch pipettes pulled from borosilicate glass capillaries (1B120F-4, World Precision Instruments). Pipette tip resistances were 4-6 $M \Omega$ for ganglion cell recordings and 5-8 M $\Omega$ for AC recordings. Patch pipettes were filled with internal solutions containing the following (in $\mathrm{mM}$ ): 120 K-methanesulfonate, 10 HEPES, 0.1 EGTA, 5 NaCl, 4 ATP-Mg, 0.4 GTP-Na $\mathrm{N}_{2}$, and 10 phosphocreatine-Tris 2 , pH 7.3, 280 mOsm for current-clamp recordings; or 120 Cs-methanesulfonate, 5 TEA-Cl, 10 HEPES, 10 BAPTA, 3 NaCl, 2 QX-314-Cl, 4 ATP-Mg, 0.4 GTP-Na , and 10 phosphocreatine-Tris 2 , $\mathrm{pH} 7.3,280 \mathrm{mOsm}$ for voltage-clamp recordings. In a subset of recordings, the internal solution was supplemented with $0.05 \%(\mathrm{w} / \mathrm{v})$ Lucifer yellow to fluorescently label cells for subsequent immunohistochemical experiments. All components of internal solutions were obtained from MilliporeSigma. Membrane potential or current was amplified (MultiClamp 700B, Molecular Devices), digitized at 5 or $10 \mathrm{kHz}$ (Digidata $1440 \mathrm{~A}$, Molecular Devices), and recorded (pClamp 10.0, Molecular Devices). During voltage-clamp recordings, excitatory or inhibitory currents were isolated by clamping near the reversal potential for chloride $\left(\mathrm{E}_{\mathrm{Cl}},-67 \mathrm{mV}\right)$ or cations $\left(\mathrm{E}_{\text {cation }}, 0 \mathrm{mV}\right)$, respectively. Series resistance (10-25 M $\Omega$ ) was compensated by $50 \%$. Voltage- and current-clamp recordings were corrected for a $-9 \mathrm{mV}$ liquid junction potential.

In most cases, specific ipRGC types (i.e., M1-M5) were targeted for patch-clamp recording using genetically encoded fluorescent reporters, which were visualized using a custom-built two-photon laser-scanning microscope controlled by ScanImage (Vidrio Technologies) (Borghuis et al., 2013). Two-photon excitation was provided by a tunable Coherent Chameleon Ultra II laser $\left(\lambda_{\text {peak }}=910 \mathrm{~nm}\right)$. Following recording, ipRGC type identity was confirmed by visualizing fluorescent dye-loaded dendritic arbors, first in live tissue (via two-photon imaging) and later in fixed tissue (via confocal imaging). M1-M3 ipRGCs were readily identified using an $O p n 4-g f p$ allele and distinguished morphologically by their unique stratification profiles within the IPL: in mice, M1 ipRGCs monostratify within the outer margin of the IPL; M2 ipRGCs monostratify 
A

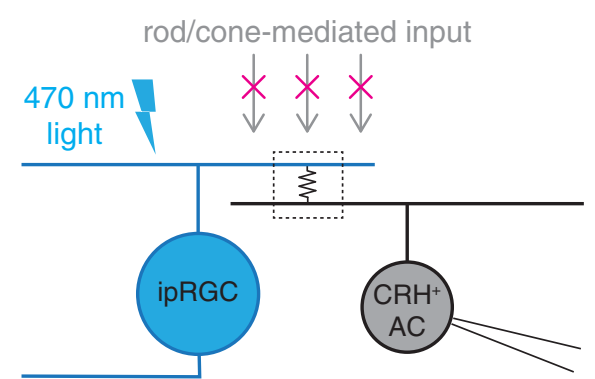

B
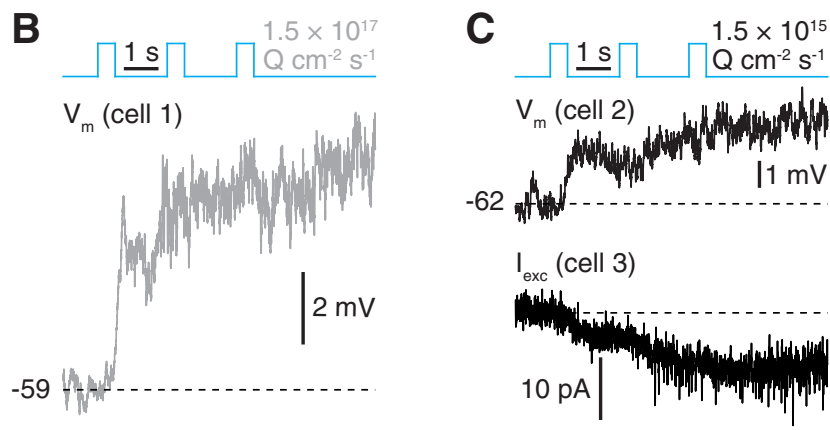

D $1.5 \times 10^{16} \mathrm{Q} \mathrm{cm}^{-2} \mathrm{~s}^{-1}$
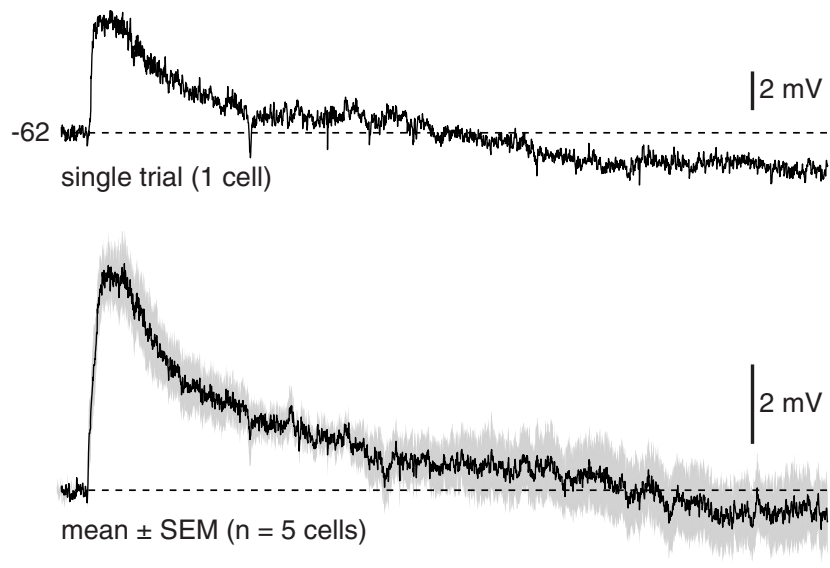

Figure 1. $\mathrm{CRH}^{+} \mathrm{ACs}$ exhibit slow, rod- and cone-independent light responses. $A$, Circuit diagram illustrating putative ipRGC-AC electrical synapse and experimental paradigm. An ipRGC participates in a gap junction-mediated electrical synapse (middle, boxed resistor symbol) with a $\mathrm{CRH}^{+} \mathrm{AC}$. During pharmacological blockade of rod- and cone-mediated input, photostimulation of the ipRGC depolarizes the coupled $\mathrm{CRH}^{+} \mathrm{AC}$. $\boldsymbol{B}$, Current-clamp recording of rod- and cone-independent light response in a $\mathrm{CRH}^{+} \mathrm{AC}$ targeted in a $\mathrm{Crh}-\mathrm{Cre}^{+/-}$; Ai14 ${ }^{+/-}$retina $\left(\Phi_{\text {stim }}=1.5 \times 10^{17} \mathrm{Q} \mathrm{cm}^{-2} \mathrm{~s}^{-1}\right)$. C, Same format as in $\boldsymbol{B}$, but for 100 -fold lower photostimulus intensity. Traces represent current-clamp (top) and voltage-clamp (bottom; $V_{\text {hold }}=-70 \mathrm{mV}$ ) recordings from separate $\mathrm{CRH}^{+} \mathrm{ACs}$. D, Rod- and cone-independent light responses to a $1 \mathrm{~s}$ light pulse in a single $\mathrm{CRH}^{+} \mathrm{AC}$ (top) and average response of $5 \mathrm{ACs}$ (bottom). Gray shading represents \pm SEM across cells.

within the inner margin of the IPL; and M3 ipRGCs bistratify within both margins of the IPL (Schmidt and Kofuji, 2009; Ecker et al., 2010; Schmidt and Kofuji, 2011) (see Fig. 5E2).

Unlabeled M4 ipRGCs were identified before recordings based on the conjunction of large soma size, characteristic of the set of " $\alpha$ " RGC types to which M4 ipRGCs belong (Estevez et al., 2012; Schmidt et al., 2014; Krieger et al., 2017) (see Fig. 5C); and sustained spike responses to the onset of a light spot $\left(\lambda_{\text {peak }}=395 \mathrm{~nm}, \sim 10^{4}\right.$ photoisomerizations cone $^{-1} \mathrm{~s}^{-1}, 400 \mu \mathrm{m}$ diameter) (see Fig. $5 E 1$ ), presented by a modified
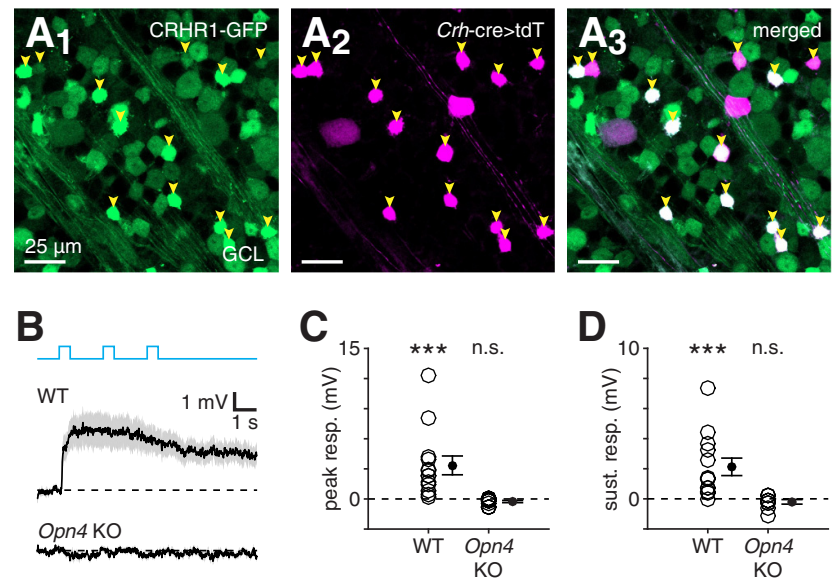

Figure 2. Rod- and cone-independent light responses of $\mathrm{CRH}^{+} \mathrm{ACs}$ depend on melanop$\sin$. $A$, Confocal micrographs showing GFP fluorescence $\left(A_{1}\right)$, tdT fluorescence driven by a Crh-cre allele $\left(\boldsymbol{A}_{2}\right)$, and their overlap $\left(\boldsymbol{A}_{3}\right)$ in the $\mathrm{GCL}$ of a $\mathrm{Crhr}^{-g f p}{ }^{+}$; $\mathrm{Crh}_{\text {-cre }}{ }^{+/-} ; \mathrm{AiT}^{+/-}$ retina. Arrowheads indicate small somas that exhibit overlap between both fluorescence channels. $\boldsymbol{B}$, Effect of melanopsin $\mathrm{KO}$ on rod- and cone-independent light responses in $\mathrm{CRH}^{+}$ ACs. Top, Black trace represents mean rod- and cone-independent voltage response of presumed CRH ${ }^{+}$ACs $(n=13)$ to photostimulation. Gray shading represents \pm SEM across cells. Bottom, Same format as top but for presumed $\mathrm{CRH}^{+} \mathrm{ACs}(n=9)$ recorded in melanopsin KO (Opn4 K0; i.e., Crhr1-gfp ${ }^{+}$;Opn4 ${ }^{\text {(re//re }}$ ) retinas $\left(\Phi_{\text {stim }}=1.5 \times 10^{17} \mathrm{Q} \mathrm{cm}^{-2} \mathrm{~s}^{-1}\right)$. C, D, Peak $(\boldsymbol{C})$ and sustained $(\boldsymbol{D})$ voltage responses in $\mathrm{CRH}^{+} \mathrm{ACS}$ shown in $\boldsymbol{B} .{ }^{* * * *} p<0.001$.

video projector focused through a sub-stage condenser lens onto the retina (Borghuis et al., 2013).

M5 ipRGCs were targeted using a Crhr1-gfp allele (Justice et al., 2008). In retinas of Crhrl-gfp ${ }^{+}$mice, putative M5 ipRGCs were initially identified by targeting intensely $\mathrm{GFP}^{+}$, moderately sized somas located in the ganglion cell layer (GCL) for loose-patch recording; such cells were further studied if they exhibited sustained spike responses to the onset of a light spot (see Fig. 5E3). Cells studied based on these properties and subsequently visualized by confocal microscopy exhibited morphological properties characteristic of M5 ipRGCs: monostratified dendritic arbors within the inner margin of the IPL, dense dendritic branching, and relatively small dendritic and somatic diameters (Stabio et al., 2018; Sonoda et al., 2020) (see Fig. 4D1). In all recorded ipRGCs, intrinsic photosensitivity was confirmed by the presence of sustained photocurrents during pharmacological blockade of rod- and conemediated input (see below).

In $\mathrm{Crh}-\mathrm{cre}^{+/-} ; \mathrm{Ail4}^{+/-}$or $\mathrm{Crh}-\mathrm{cre}^{+/-} ; \mathrm{A} i 32^{+/-}$mice, putative $\mathrm{CRH}^{+}$ ACs (also known as CRH-1 ACs) (Zhu et al., 2014; Jacoby et al., 2015; Park et al., 2018) were identified by their small fluorescent somas in the GCL. During whole-cell recording, $\mathrm{CRH}^{+}$AC identity was confirmed by nonspiking physiology and medium-field ( $\sim 250-\mu \mathrm{m}$-diameter), highly branching neuritic morphology (Zhu et al., 2014; Jacoby et al., 2015; Park et al., 2018), as visualized in real time using two-photon fluorescence excitation of pipette-loaded Lucifer yellow. These physiological and morphological properties unambiguously distinguished $\mathrm{CRH}^{+} /$ CRH-1 ACs from rare CRH-2 and CRH-3 ACs, which exhibit spiking and wide-field ( $>1$-mm-diameter) morphology; and from rare GCs, which include transient OFF $\alpha$ GCs and GCs with asymmetric dendritic arbors that stratify near the outer margin of the IPL (Zhu et al., 2014; Park et al., 2018). In Crhrl-gfp mice, putative $\mathrm{CRH}^{+}$ACs were targeted by locating small, intensely $\mathrm{GFP}^{+}$somas in the GCL, followed by confirmation of physiological and morphological criteria described above. For a subset of $\mathrm{CRH}^{+}$ACs recorded and dye-filled in Crhr1-gfp mice, neuritic morphology was visualized in fixed tissue to confirm stratification within the inner margin of the IPL, as is characteristic of $\mathrm{CRH}^{+} \mathrm{ACs}$ (Zhu et al., 2014; Jacoby et al., 2015; Park et al., 2018).

During all recordings, melanopsin- and/or ChR2-mediated responses were evoked by an LED ( $\lambda_{\text {peak }}=470 \mathrm{~nm}$; M470L3, Thorlabs) projected through the aperture (400 $\mu \mathrm{m}$ diameter) of an iris diaphragm (CP20S, Thorlabs), driven by a T-Cube LED driver (LEDD1B, Thorlabs), and 
focused through a substage condenser lens onto the retina. The maximum light intensity $\left(\Phi_{\max }\right)$ at the sample plane was $4.8 \times 10^{17}$ quanta $(\mathrm{Q}) \mathrm{cm}^{-2} \mathrm{~s}^{-1}$, and neutral density filters were inserted into the light path to attenuate stimulus intensity by 10 - or 100 -fold. Stimuli were $\gamma$-corrected to account for a nonlinear relationship between voltage input to the LED driver and light output of the LED, as measured at the sample plane. During all experiments, conventional photoreceptor-mediated input was pharmacologically blocked via bath application of the following standard drug cocktail (in $\mu \mathrm{M}$ ): 50 D-AP5 (Alomone Labs), 50 DNQX (Alomone Labs), 20 L-AP4 (Alomone Labs), and 2 ACET (Tocris) (Park et al., 2015; Park et al., 2018; Pottackal et al., 2020). For experiments designed to block gap junctions, the standard solution was supplemented with $100 \mu \mathrm{M}$ meclofenamic acid (MFA, Tocris Bioscience). For experiments designed to isolate coupling currents in ipRGCs during optogenetic stimulation of $\mathrm{CRH}^{+}$ACs, the standard solution was supplemented with $25 \mu \mathrm{M}$ SR-95531 (Alomone Labs) to eliminate unclamped IPSCs mediated by $\mathrm{GABA}_{\mathrm{A}}$ receptors. For experiments designed to block L-type VGCCs, the standard solution was supplemented with $30 \mu \mathrm{m}$ isradipine (Abcam).

Linear-nonlinear cascade analysis. Linear-nonlinear (LN) cascade analysis was performed as described in detail previously (Jarsky et al., 2011; Pottackal et al., 2020). Briefly, quasi-white-noise (WN) stimuli consisted of 10 consecutive $10 \mathrm{~s}$ trials that each included $7.5 \mathrm{~s}$ of a unique stimulus sequence followed by $2.5 \mathrm{~s}$ of a repeated stimulus sequence. For each recorded cell, responses to unique stimuli were used in LN model construction, whereas responses to repeated stimuli were used in evaluating model accuracy. WN stimuli were ideally low-pass filtered at $30 \mathrm{~Hz}$. For each cell, a linear filter was computed from cross-correlation of the WN stimulus with the recorded IPSC. The filter was then convolved with the stimulus to generate a linear prediction of the response, which was then plotted against the recorded response. Plotted points were equally divided into 100 bins along the linear prediction axis. Points within each bin were averaged along both dimensions (i.e., predicted response vs recorded response), generating 100 points that were then fit with a Gaussian cumulative distribution function $N(x)$; this served as the static nonlinearity component of the LN model. The linear prediction was passed through this static nonlinearity to generate the final LN model output. The accuracy of the LN model was quantified as the squared Pearson correlation coefficient $\left(r^{2}\right)$ between (1) the model response to the repeated stimulus and (2) the mean of 10 recorded responses to the repeated stimulus. To quantify the linearity of each modeled recording, a rectification index $i_{\text {rect }}$ was computed from the static nonlinearity $N(x)$ as follows:

$$
i_{\text {rect }}=\frac{\left|N\left[\max \left(r_{L[b i n]}\right)\right]+N\left[\min \left(r_{L[b i n]}\right)\right]-2 N(0)\right|}{N\left[\max \left(r_{L[b i n]}\right)\right]-N\left[\min \left(r_{L[b i n]}\right)\right]}
$$

where $r_{L[b i n]}$ is the set of 100 values obtained after binning and averaging along the linear prediction axis.

Tracer/dye injection. Neurobiotin (Vector Laboratories) was injected into ipRGCs using a loose-patch electroporation protocol similar to that described previously (Pérez de Sevilla Müller et al., 2010). Briefly, 4\% Neurobiotin and $0.1 \%$ Lucifer yellow (MilliporeSigma) were added to the current-clamp solution. To minimize the possibility of nonspecific uptake of Neurobiotin from the extracellular space, positive pressure was not applied to Neurobiotin-filled patch pipettes. Loose-seals (10-15 M $\Omega$ ) were initially obtained from targeted somas, followed by brief application of $-20 \mathrm{nA}$ inward current pulses at $10 \mathrm{~Hz}$, which were terminated immediately following visualization of Lucifer yellow within the soma. Subsequently, the direction of current injection was reversed and the amplitude reduced to $8-15 \mathrm{nA}$, and $10 \mathrm{~Hz}$ current pulses were administered continuously for $12-20 \mathrm{~min}$. For most injections, the retina was exposed to bright light $\left(\sim 10^{4}\right.$ photoisomerizations cone $\left.\mathrm{e}^{-1} \mathrm{~s}^{-1}\right)$ for $\sim 1 \mathrm{~h}$ following the final injection to promote increased gap junctional coupling strength (Hu et al., 2010; Nath and Schwartz, 2017). For a subset of experiments to study morphological development of $\mathrm{CRH}^{+} \mathrm{AC}$ neuritic arbors, $0.7 \%$ Lucifer yellow in distilled water was injected into $\mathrm{CRH}^{+} \mathrm{AC}$ somas using a briefer variant of this electroporation protocol. Following loose-seal formation, $-20 \mathrm{nA}$ inward current pulses were applied at $10 \mathrm{~Hz}$ until initial visualization of dye loading. Subsequently, current pulse amplitude was reduced to $-10 \mathrm{nA}$, and injection was resumed for 3-6 s.

Histology. For cells filled with Lucifer yellow during whole-cell recording or injected with Neurobiotin, or for fluorescence colabeling experiments, dissected retinas were fixed in PFA ( $4 \% \mathrm{v} / \mathrm{v}$ in PBS) for $\sim 1 \mathrm{~h}$ at room temperature and subsequently stored in $\mathrm{PBS}$ at $4^{\circ} \mathrm{C}$. During immunohistochemistry, whole-mount retinas were incubated with $6 \%$ normal donkey serum (017-000-121, Jackson ImmunoResearch Laboratories, RRID:AB_2337258) and 0.5\% Triton X-100 (T8787, MilliporeSigma) for $1 \mathrm{~h}$ at room temperature; primary antibodies, $2 \%$ normal donkey serum, and $0.5 \%$ Triton $\mathrm{X}-100$ for $\sim 1$ or $7 \mathrm{~d}$ at $4^{\circ} \mathrm{C}$; and secondary antibodies, $2 \%$ normal donkey serum, and $0.5 \%$ Triton $\mathrm{X}-100$ for $2 \mathrm{~h}$ at room temperature. Primary antibodies were diluted and incubated as follows: goat anti-ChAT, 1:200 for $\sim 1 \mathrm{~d}$ (AB144P, MilliporeSigma, RRID:AB_2079751); guinea pig anti-RBPMS, 1:1000 for $7 \mathrm{~d}$ (RBPMS1832, PhosphoSolutions, RRID:AB_2492226); rabbit anti-Lucifer yellow, 1:2000 for $\sim 1 \mathrm{~d}$ (A-5750, Thermo Fisher Scientific, RRID:AB 2536190); rabbit anti-melanopsin, 1:100 for $\sim 1 \mathrm{~d}$ (AB-N39, Advanced Targeting Systems, RRID:AB_1608076); and mouse anti-neurofilament H (SMI-32), 1:500 for 2 d (SMI-32R, BioLegend, RRID:AB_509997). Secondary antibodies (diluted at 1:500) were donkey anti-goat IgG conjugated to AlexaFluor-633 (A-21082, Thermo Fisher Scientific, RRID:AB_2535739), donkey anti-rabbit IgG conjugated to Cy3 (711-165-152, Jackson ImmunoResearch Laboratories, RRID:AB 2307443), donkey anti-guinea pig IgG conjugated to Cy5 (706-175148, Jackson ImmunoResearch Laboratories, RRID:AB_2340462), donkey anti-rabbit IgG conjugated to Cy5 (711-175-152, Jackson ImmunoResearch Laboratories, RRID:AB_2340607), and donkey antimouse IgG conjugated to Cy5 (715-175-150, Jackson ImmunoResearch Laboratories, RRID:AB_2340819). For experiments to visualize and/or quantify overlap between fluorescently labeled ganglion cell populations, RBPMS was used as a marker of ganglion cell identity (Rodriguez et al., 2014) (see Fig. 4B).

Confocal $z$-stack images of filled dendritic (GCs) or neuritic (ACs) arbors were obtained by a Carl Zeiss LSM 800 laser scanning microscope equipped with a $20 \times$ air objective $(\mathrm{NA}=0.8)$. All neuritic arbor measurements, fluorescence intensity measurements, and cell counts were performed using Image). For each $\mathrm{CRH}^{+} \mathrm{AC}$, the area of its arbor was measured by manually drawing the smallest convex polygon that contained the entire $z$-projected arbor and then measuring the area of this polygon; arbor diameter was subsequently computed as the diameter of the circle whose area equals that of the polygon. Neurite length was measured by manually tracing neurites using the Simple Neurite Tracer plugin and then summing the path lengths of all neurite segments within the arbor.

For experiments to quantify GFP fluorescence intensity in identified ipRGC subpopulations, $z$-stack images of the GCL were obtained using a $20 \times$ air objective $(\mathrm{NA}=0.8)$. In each condition, for at least one retina from each of $2 \mathrm{Opn} 4^{\mathrm{Cre} /+} ; \mathrm{Ail1}^{+/-} ; \mathrm{Crhrl}-\mathrm{gfp}{ }^{+}$mice, up to four $607 \mu \mathrm{m} \times 607 \mu \mathrm{m}$ regions were imaged $\sim 1-1.5 \mathrm{~mm}$ from the optic disk. Polygonal ROIs were manually drawn around somas of imaged ipRGCs, which were identified by Cre-dependent tdT expression. For each image, an additional ROI was drawn around a patch of GFP-negative cells to measure background fluorescence. For each ROI, mean pixel intensity and total area were measured, with the latter used to compute soma diameter as above for arbor diameter. The background-subtracted intensity measured for each ipRGC soma was then normalized by the median absolute deviation of the sample to enable multiple image samples to be merged. For experiments to count overlap between fluorescently labeled cell populations, $z$-stack images of the GCL were obtained using a $40 \times$ oil objective $(\mathrm{NA}=1.4)$. In each condition, for at least one retina in each of 2 or more mice, up to eight $159 \mu \mathrm{m} \times 159 \mu \mathrm{m}$ regions were imaged $\sim 1-1.5$ $\mathrm{mm}$ from the optic disk. Cell counts were obtained using the Cell Counter plugin in ImageJ.

Experimental design and statistical analysis. Consistent with comparable studies and conventions in the field, each group included 4-13 cells recorded from at least 2 mice of either sex. Summary values are reported as mean \pm SEM. Statistical comparisons were performed using 
A

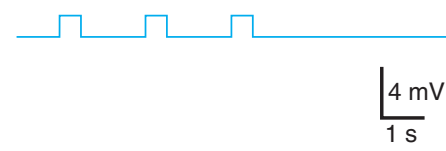

P9-10
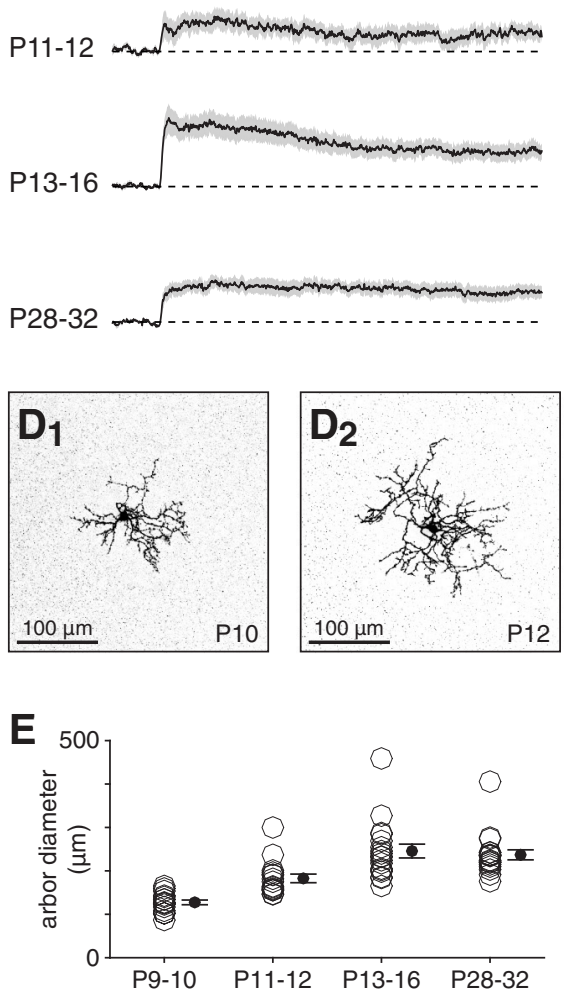
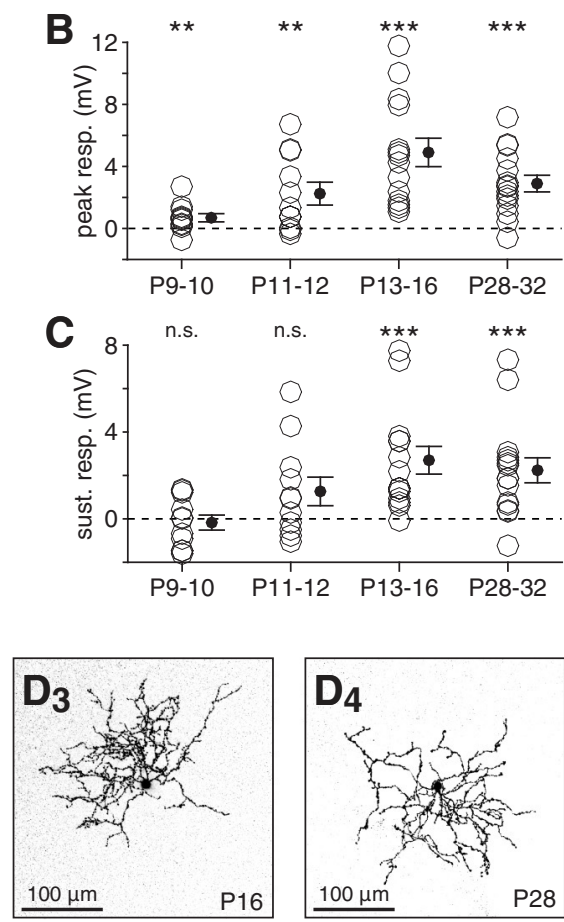

$\mathbf{F}$

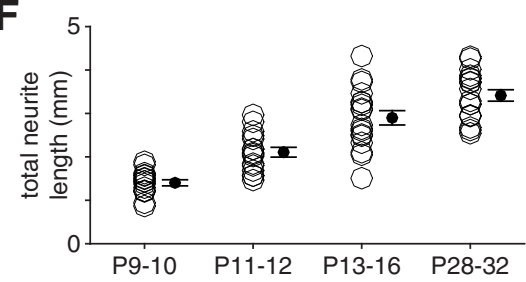

Figure 3. Melanopsin-dependent light responses of $\mathrm{CRH}^{+} \mathrm{ACs}$ mature after eye opening. $\boldsymbol{A}$, Melanopsin-dependent voltage responses of $\mathrm{CRH}^{+} \mathrm{ACS}$ at four developmental time points. From top, mean (black) \pm SEM (gray) of melanopsin-dependent light responses across $\mathrm{CRH}^{+}$ACs at P9-P10 $(n=11), \mathrm{P} 11-\mathrm{P} 12(n=11), \mathrm{P} 13-\mathrm{P} 16(n=14)$, and P28-P32 $(n=15)$ $\left(\Phi_{\text {stim }}=1.5 \times 10^{17} \mathrm{Q} \mathrm{cm}^{-2} \mathrm{~s}^{-1}\right) . \boldsymbol{B}, \boldsymbol{C}$, Peak $(\boldsymbol{B})$ and sustained $(\boldsymbol{C})$ voltage responses in $\mathrm{CRH}^{+} \mathrm{ACs}$ shown in $\boldsymbol{A}$. $\boldsymbol{D}$, Confocal micrographs showing morphological development of $\mathrm{CRH}^{+} \mathrm{AC}$ neurites at P10 $\left(\boldsymbol{D}_{1}\right)$, P12 $\left(\boldsymbol{D}_{2}\right)$, P16 $\left(\boldsymbol{D}_{3}\right)$, and P28 $\left(\boldsymbol{D}_{4}\right)$. Images are projections of $\sim 20$ consecutive slices of a $Z$ stack $(0.41 \mu \mathrm{m}$ spacing). $\boldsymbol{E}, \boldsymbol{F}$, Neurite arbor diameter $(\boldsymbol{E})$ and total neurite length $(\boldsymbol{F})$ of $\mathrm{CRH}^{+} \mathrm{ACS}(n=16, n=16, n=18$, and $n=18)$ at time points studied in $A-C .{ }^{* *} p<0.01$. ${ }^{* * *} p<0.001$.

nonparametric tests in most cases, with all exceptions indicated. Onesided statistical tests were performed for comparisons in which specific null hypotheses were tested: (1) that light does not evoke depolarization in $\mathrm{CRH}^{+} \mathrm{ACs}$ in WT or melanopsin KO conditions (see Fig. 2C,D); (2) that light does not evoke depolarization in $\mathrm{CRH}^{+}$ACs at each developmental time point tested (see Fig. 3B,C); (3) that light not does not evoke inward currents in each ipRGC type during optogenetic stimulation of $\mathrm{CRH}^{+}$ACs (see Fig. 6E); (4) that MFA does not decrease melanopsinmediated depolarization of M5 ipRGCs (see Fig. 9B); (5) that $\mathrm{CRH}^{+} \mathrm{AC}$ photocurrent amplitude does not decrease from a holding potential of $-70 \mathrm{mV}\left(\sim \mathrm{E}_{\mathrm{Cl}}\right)$ to a holding potential of $0 \mathrm{mV}$ ( $\left.\mathrm{E}_{\text {cation }}\right)$ (see Fig. $\left.9 D\right)$; and (6) that high-frequency noise does not increase in outward currents recorded in each ipRGC type during optogenetic stimulation of $\mathrm{CRH}^{+}$ ACs (see Fig. 10E). The Tukey-Kramer method (see Fig. 3E,F) or Bonferroni correction (see Fig. $6 F$ ) was used to control for multiple comparisons. Exact $p$ values are reported up to $p<0.001$.

\section{Results}

\section{$\mathrm{CRH}^{+}$ACs exhibit slow, rod- and cone-independent light responses}

Within the IPL of the mouse retina, neurites of nonspiking $\mathrm{CRH}^{+}$ACs (also known as CRH-1 ACs) costratify extensively with dendrites of all ipRGC types, except for M1 (Zhu et al., 2014; Jacoby et al., 2015; Park et al., 2018), positioning these neurons to potentially receive electrical synaptic input from ipRGCs (Reifler et al., 2015; Sabbah et al., 2017). To test whether $\mathrm{CRH}^{+}$ACs exhibit light responses consistent with ipRGC coupling, we recorded membrane potential $\left(V_{\mathrm{m}}\right)$ in $\mathrm{CRH}^{+}$ACs in whole-mount retinas and presented light stimuli during pharmacological blockade of rod- and cone-mediated input (Fig. 1A) (Park et al., 2018). Indeed, under these conditions, strong photostimulation $\left(\Phi_{\text {stim }}=1.5 \times 10^{17}\right.$ quanta $\left.\mathrm{cm}^{-2} \mathrm{~s}^{-1}\right)$ evoked slow, rod- and cone-independent depolarization in $\mathrm{CRH}^{+}$ACs that persisted for seconds following stimulus offset (amplitude $3 \mathrm{~s}$ after offset $=6.2 \pm 1.1 \mathrm{mV}$, $n=8, p=0.008, W=36$, Wilcoxon signedrank test; Fig. $1 B$ ). We observed similarly prolonged rod- and cone-independent depolarization and inward currents in $\mathrm{CRH}^{+} \mathrm{ACs}$ at 100-fold lower photostimulus intensity (Fig. 1C). Depolarizing responses to a single $1 \mathrm{~s}$ light pulse of intermediate intensity decayed to baseline $>20$ s after stimulus offset on average ( $n=5$; Fig. $1 D)$, exhibiting decay kinetics similar to those of non-M1 ipRGC intrinsic photoresponses (Schmidt and Kofuji, 2009; Ecker et al., 2010; Estevez et al., 2012; Stabio et al., 2018; Sonoda et al., 2018).

\section{Rod- and cone-independent light responses in $\mathrm{CRH}^{+}$ACs depend on melanopsin}

We next tested whether rod- and cone-independent light responses in $\mathrm{CRH}^{+} \mathrm{ACs}$ depend on melanopsin by generating a mouse line in which melanopsin was deleted and $\mathrm{CRH}^{+}$ACs could be targeted for recording. To do so, we crossed Crhrl-gfp mice (Justice et al., 2008), in which $\mathrm{CRH}^{+}$ACs strongly express GFP and can be routinely targeted for recording, to Opn4-cre knockin mice, for which the Opn $4^{\mathrm{Cre} / \mathrm{Cre}}$ genotype results in melanopsin $\mathrm{KO}$ (Ecker et al., 2010) (see Materials and Methods). In Crhrl-gfp mice, GFP expression is driven by regulatory elements of Crhrl, which encodes CRH receptor 1 (CRHR1), the primary CRH receptor (Justice et al., 2008) (see Materials and Methods).

First, we validated the Crhrl-gfp line by generating Crhrlgfp ${ }^{+}$; Crh-cre ${ }^{+/-} ; \mathrm{Ail4}^{+/-}$mice and observing that all $\mathrm{CRH}^{+}$ ACs (68 of 68 cells, $n=2$ mice) in which Cre recombinase drove tdT expression also expressed GFP, with most exhibiting intense GFP expression (Fig. 2A). Consistent with $\mathrm{CRH}^{+}$ AC identity, current-clamp recordings from small, intensely $\mathrm{GFP}^{+}$somas in Crhrl-gfp retinas during photostimulation revealed significant rod- and cone-independent depolarization (peak response: $3.3 \pm 0.9 \mathrm{mV}, n=13, p<0.001, W=91$; sustained response [3 s after offset]: $2.1 \pm 0.6 \mathrm{mV}, n=13$, $p<0.001, W=90$, one-sided Wilcoxon signed-rank tests; Fig. $2 B$ ), which was qualitatively similar to that recorded in 
$\mathrm{tdT}^{+} \mathrm{CRH}^{+} \mathrm{ACs}$ targeted in $\mathrm{Crh}-\mathrm{cre}^{+/-} ; \mathrm{Ai14}^{+/-}$retinas (Fig. $1 B$ ). To explicitly test whether rod- and cone-independent light responses in $\mathrm{CRH}^{+}$ACs depend on melanopsin, we recorded from retinas of $\mathrm{Crhr} 1-g f p^{+} ; \mathrm{Opn} 4^{\mathrm{Cre} / \mathrm{Cre}}$ mice and found that photostimulation failed to evoke rod- and coneindependent depolarization in $\mathrm{CRH}^{+}$ACs (peak response: $-0.3 \pm 0.1 \mathrm{mV}, n=9, p=0.963, W=5$; sustained response: $-0.2 \pm 0.2 \mathrm{mV}, n=9, p=0.898, W=12$, one-sided Wilcoxon signed-rank tests; Fig. 2C). These results demonstrate that rod- and cone-independent light responses in $\mathrm{CRH}^{+} \mathrm{ACs}$ depend on melanopsin and, moreover, suggest that these responses are generated by ipRGCs.

\section{Melanopsin-dependent light responses in $\mathrm{CRH}^{+} \mathrm{ACs}$ strengthen following rod and cone integration}

During postnatal development, ipRGCs can drive light responses in non-ipRGCs, including ACs, before rods and cones functionally mature and integrate into retinal circuitry around P12 (Sekaran et al., 2005; Tu et al., 2005; Kirkby and Feller, 2013; Arroyo et al., 2016; Caval-Holme et al., 2019). Therefore, we examined the development of melanopsin-dependent depolarization in $\mathrm{CRH}^{+} \mathrm{ACs}$ by measuring these light responses at four time points: postnatal days 9-10 (P9-P10), P11-P12, P13-P16, and P28-P32. We observed small but significant peak depolarization at P9-P10 $(0.7 \pm 0.3 \mathrm{mV}, n=11, p=0.009, W=59)$ and larger peak responses at all subsequent time points (P11-P12: $2.3 \pm 0.7 \mathrm{mV}$, $n=11, p=0.005, W=61$; P13-P16: $4.9 \pm 0.9 \mathrm{mV}, n=14, p<0.001$, $W=105$; P28-P32: $2.9 \pm 0.5 \mathrm{mV}, n=15, p<0.001, W=118$, onesided Wilcoxon signed-rank tests; Fig. $3 A, B)$. However, depolarizing responses at P9-P10 and P11-P12 did not exhibit significant sustained components (P9-P10: $-0.2 \pm 0.3 \mathrm{mV}, n=11, p=0.319$, $W=27$; P11-P12: $1.3 \pm 0.7 \mathrm{mV}, n=11, p=0.062, W=51)$, in contrast to responses at both later time points (P13-P16: $2.7 \pm 0.6 \mathrm{mV}$, $n=14, p<0.001, W=104$; P28-P32: $2.2 \pm 0.6 \mathrm{mV}, n=15, p<$ $0.001, W=115$, one-sided Wilcoxon signed-rank tests; Fig. $3 A, C)$. These results suggest that, while melanopsin-dependent light responses of $\mathrm{CRH}^{+}$ACs may begin developing just before outer photoreceptor integration and eye opening, functional coupling strengthens after these events. Thus, ipRGC-mediated input to $\mathrm{CRH}^{+}$ACs operates primarily in tandem with conventional rodand cone-mediated input in the mature retinal circuit.

Next, we examined whether the morphological development of $\mathrm{CRH}^{+} \mathrm{AC}$ neuritic arbors parallels the observed development of melanopsin-dependent light responses in $\mathrm{CRH}^{+} \mathrm{ACs}$. Morphological analysis of dye-loaded $\mathrm{CRH}^{+}$ACs at the same time points revealed changes in arbor diameter $(p<0.001$, $F_{(3,64)}=21.4$, one-way ANOVA) and total neurite length $\left(p<0.001, F_{(3,64)}=47.3\right.$, one-way ANOVA $)$ across the studied time points. Specifically, we observed increases in arbor diameter from P9-P10 $(127.9 \pm 5.4 \mu \mathrm{m}, n=16)$ and P11-P12 $(182.5 \pm 9.9 \mu \mathrm{m}, n=16)$ to all later time points (P13-P16: $245.2 \pm 15.9 \mu \mathrm{m}, n=18 ; \mathrm{P} 28-\mathrm{P} 32: 236.8 \pm 11.5 \mu \mathrm{m}, n=18$; P9-P10 vs P11-P12: $p=0.011$; P9-P10 vs P13-P16 and P28P32: $p<0.001$; P11-P12 vs P13-P16: $p=0.002$; P11-P12 vs P28-P32: $p=0.009$, Tukey-Kramer tests; Fig. 3D,E). Likewise, we observed increases in total neurite length from P9-P10 $(1402.7 \pm 70.2 \mu \mathrm{m})$ and P11-P12 $(2107.2 \pm 112.8 \mu \mathrm{m})$ to all later time points (P13-P16: 2899.5 $\pm 165.1 \mu \mathrm{m}$; P28-P32: $3414.1 \pm 131.7 \mu \mathrm{m}$; P9-P10 vs P11-P12: $p=0.002$; P9-P10 vs P13-P16 and P28-P32: $p<0.001$; P11-P12 vs P13-P16 and P28-P32: $p<0.001$, Tukey-Kramer tests; Fig. $3 D, F)$. Thus, in $\mathrm{CRH}^{+}$ACs, the development of melanopsin-dependent light responses proceeds with a time course similar to that of neuritic morphology. It is important to emphasize, however, that neither the development of $\mathrm{CRH}^{+} \mathrm{AC}$ morphology nor that of outer photoreceptor connectivity necessarily has a causal relationship with the development of melanopsin-dependent light responses in $\mathrm{CRH}^{+}$ACs. Nonetheless, these results show that melanopsin-dependent light responses of $\mathrm{CRH}^{+} \mathrm{ACs}$ achieve full functional strength only after outer photoreceptor integration, suggesting that this ipRGC signaling pathway functions primarily from late postnatal development through maturity.

\section{Identification of a novel transgenic mouse line for targeting specific ipRGC types}

To study functional connectivity between $\mathrm{CRH}^{+}$ACs and specific ipRGC types, we used transgenic mice in which specific subsets of ipRGC types can be efficiently targeted for recording (see Materials and Methods). M1-M3 ipRGCs, which most strongly express melanopsin, can be targeted using an Opn4-gfp allele, while unlabeled M4 ipRGCs can be targeted based on the conjunction of their large soma size and sustained spike responses to light onset (Estevez et al., 2012; Krieger et al., 2017; Park et al., 2018). Additionally, we sought a transgenic mouse line to facilitate targeting of other ipRGC types reported in mice (i.e., M5 and/or M6) (Ecker et al., 2010; Stabio et al., 2018; Quattrochi et al., 2019).

Consequently, we generated Crhr1-gfp ${ }^{+} ; \mathrm{Opn} 4^{\mathrm{Cre} /+} ; \mathrm{Ail}^{+/-}$ mice, in which all ipRGCs express tdT, to visualize overlap between $\mathrm{GFP}^{+}$cells and all ipRGC types. In these mice, $86.2 \%$ (382 of $443, n=2$ mice) of all $\mathrm{tdT}^{+}$somas in the GCL also expressed GFP, while $20.4 \%$ (382 of 1872) of all $\mathrm{GFP}^{+}$RGC somas also expressed tdT (Fig. 4A,B). RGCs were identified in these retinas by immunostaining against RBPMS (Rodriguez et al., 2014). Across all ipRGCs, somatic GFP fluorescence intensity exhibited a distribution with a highly positive skew, indicating a fraction of intensely GFP ${ }^{+}$ipRGCs (Fig. 4C); the fluorescence intensity of these ipRGCs was exceeded only by that of $\mathrm{CRH}^{+} \mathrm{ACs}$ (Fig. 2A). When targeted for recording and dye-filling, such cells exhibited both small, slowly decaying intrinsic photocurrents; and dendritic arbors with small diameters and highly branched morphology (Fig. 4D). These properties are consistent with M5 ipRGC identity (Stabio et al., 2018) but not unambiguously indicative of it (Sonoda et al., 2020).

To determine whether M5 ipRGCs types are reliably identifiable as intensely $\mathrm{GFP}^{+}$cells in Crhr1-gfp retinas, we genetically labeled all ipRGCs while immunohistochemically labeling specific ipRGC subpopulations. We first examined the possibility that M4 ipRGCs, a subset of which exhibit arbor diameters similar to those of M5 ipRGCs (Sonoda et al., 2020), comprise at least some of the intensely $\mathrm{GFP}^{+}$ipRGCs. To do so, we immunostained retinas of Crhrl-gfp ${ }^{+} ; \mathrm{Opn} 4^{\mathrm{Cre} /+} ; \mathrm{Ail4}^{+/-}$mice with SMI32 , an antibody against neurofilament $\mathrm{H}$ that strongly labels M4 ipRGCs as well as up to three other " $\alpha$ " RGC types (Bleckert et al., 2014; Krieger et al., 2017; Sonoda et al., 2020). In these retinas, M4 ipRGC somas were readily identified by the conjunction of Cre-dependent $\mathrm{tdT}$ expression, indicating ipRGC identity; and SMI-32 staining, indicating $\alpha$ RGC identity (Fig. 5A). Typical of $\alpha$ RGCs, M4 ipRGCs identified using these criteria exhibited much larger soma diameters than nonM4 ipRGCs, with minimal overlap between the corresponding distributions $(p<0.001, D=0.95$, Kolmogorov-Smirnov test; Fig. 5C). Notably, M4 ipRGCs consistently exhibited weak-tointermediate GFP fluorescence, whereas the fluorescence intensity distribution of non-M4 ipRGCs exhibited a highly positive skew ( $p<0.001, D=0.43$, Kolmogorov-Smirnov test; 

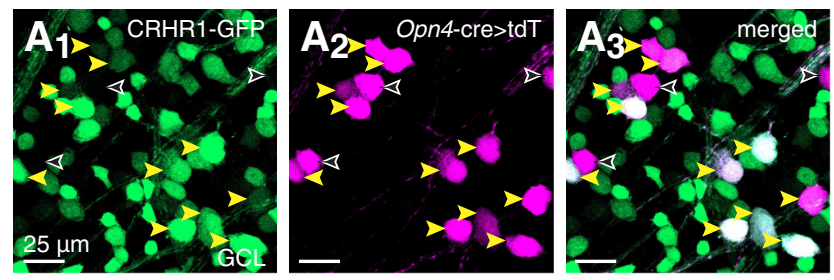

B

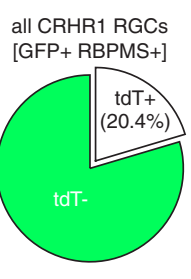

all ipRGCs

$[\mathrm{tdT}+(\mathrm{RBPMS}+)]$

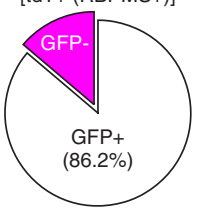

C
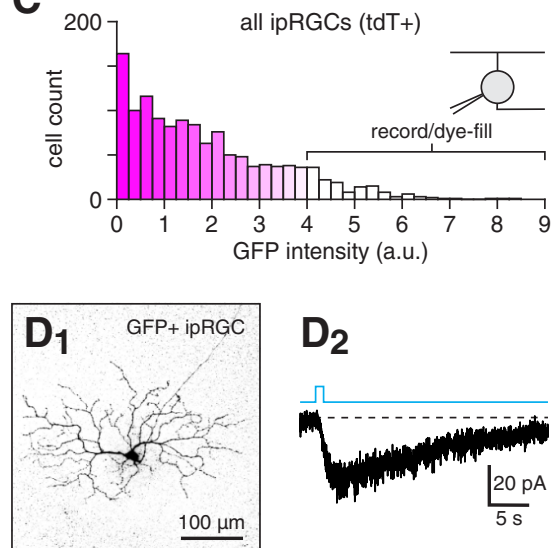

Figure 4. Labeling of ipRGCs in Crhr1-gfp retinas. A, GFP labeling of a subset of genetically identified ipRGCs in a Crhr1-gfp ${ }^{+} ; O p n 4^{\text {(re/+ }} ; A i 14^{+/-}$retina. Confocal micrographs show GFP fluorescence (green, $\boldsymbol{A}_{7}$ ), tdT expression driven by an Opn4-cre allele (Opn4cre $>$ tdT) (magenta, $\left.A_{2}\right)$, and their overlap $\left(A_{3}\right)$ in the $\mathrm{GCL}$ of a $\mathrm{Crhr1}_{\text {-gfp }}{ }^{+} ; \mathrm{Opn} 4^{\text {(re/+ }}$; $\mathrm{Ai14}^{+/-}$retina. Yellow arrowheads indicate somas that exhibit overlap between both fluorescence channels. Empty arrowheads indicate $\mathrm{tdT}^{+}$somas that lack GFP expression. $\boldsymbol{B}$, Quantification of overlap between genetically labeled ipRGCs $\left(\mathrm{tdT}^{+}\right)$and $\mathrm{GFP}^{+} \mathrm{RGCS}$ in the

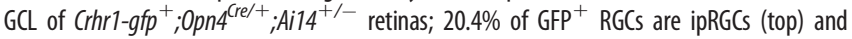
$86.2 \%$ of ipRGCs are GFP ${ }^{+}$(bottom). C, GFP fluorescence intensity distribution of genetically labeled ipRGCS (tdT $\left.{ }^{+}\right)$in $\mathrm{Crhr1}^{-g f p^{+}} ; \mathrm{Opn4^{( \textrm {re } / + }} ; \mathrm{Ail1}^{+/-}$retinas. D, Targeted recording and dye-filling of intensely GFP ${ }^{+}$ipRGCs in Crhr1-gfp retinas. $\boldsymbol{D}_{1}$, Confocal micrograph of an intensely $\mathrm{GFP}^{+}$ipRGC ( $>$90th percentile in distribution shown in $C$ ) dye-filled during whole-cell recording in a whole-mount Crhr1-gfp retina. $\boldsymbol{D}_{2}$, Melanopsin-mediated intrinsic photocurrent of cell shown in $\boldsymbol{D}_{1}$ in response to a $1 \mathrm{~s}$ light pulse $\left(\Phi_{\text {stim }}=1.5 \times 10^{16} \mathrm{Q}\right.$ $\left.\mathrm{cm}^{-2} \mathrm{~s}^{-1}\right)$.

Fig. $5 C, D)$. Thus, the subpopulation of intensely $\mathrm{GFP}^{+}$ ipRGCs systematically excludes M4 ipRGCs.

Similarly, we sought to test whether intensely $\mathrm{GFP}^{+}$ ipRGCs might include M1, M2, or M3 ipRGCs. Among ipRGCs, M1-M3 types exhibit relatively strong melanopsin immunoreactivity, whereas M4-M6 types exhibit weak or undetectable melanopsin immunoreactivity (Berson et al., 2010; Ecker et al., 2010; Estevez et al., 2012; Stabio et al., 2018; Quattrochi et al., 2019). Consequently, we immunostained against melanopsin in $\mathrm{Crhrl}-\mathrm{gfp} \mathrm{p}^{+} ; \mathrm{Opn} 4^{\mathrm{Cre} /+} ; \mathrm{Aill}^{+/-}$ retinas to assess overlap between detectably melanopsin-immunoreactive ipRGCs and $\mathrm{GFP}^{+}$cells. We found that, compared with melanopsin-immunonegative ipRGCs, melanopsin-immunopositive ipRGCs exhibited weak GFP fluorescence $(p<0.001$, $D=0.44$, Kolmogorov-Smirnov test; Fig. $5 B, D$ ), indicating that intensely $\mathrm{GFP}^{+}$ipRGCs consist primarily of ipRGC types that do not reliably exhibit strong melanopsin immunoreactivity (i. e., M4-M6). Combined with the results of our SMI-32 immunostaining experiments, these results further suggest that intensely $\mathrm{GFP}^{+}$ipRGCs in Crhr1-gfp retinas are predominantly M5 and M6 ipRGCs. Indeed, although such ipRGCs typically exhibited compact, monostratified dendritic arbors that stratified near the inner margin of the IPL (i.e., arbors consistent with M5 ipRGC identity; Fig. 4D1), we observed two M5 ipRGC-like cells that extended small fractions of their dendrites outward to stratify near the outer margin of the IPL (data not shown). The bistratified morphology of these cells partially resembled that of M6 ipRGCs (Quattrochi et al., 2019); however, because we encountered such cells rarely, we omitted them from subsequent experiments and analyses.

The identification of the Crhrl-gfp mouse as a tool for reliably targeting M5 ipRGCs enabled us to compile an ensemble of strategies for selectively targeting M1-M5 ipRGC types (Fig. 5E; see Materials and Methods). In the following experiments, we use these targeting strategies to investigate the electrical and chemical synaptic connectivity between $\mathrm{CRH}^{+} \mathrm{ACs}$ and specific ipRGC types.

\section{$\mathrm{CRH}^{+}$ACs primarily participate in electrical synapses with M5 ipRGCs}

Next, we aimed to determine whether and, if so, which specific ipRGC types participate in electrical synapses with $\mathrm{CRH}^{+} \mathrm{ACs}$. To do so, we generated mice in which $\mathrm{CRH}^{+}$ACs express ChR2-EYFP and at least one ipRGC type could be readily targeted for whole-cell voltage-clamp recording (Fig. 6A; see Materials and Methods). We predicted that ChR2 photocurrents generated in $\mathrm{CRH}^{+}$ACs would drive fast, inward currents in electrically coupled ipRGCs. During these experiments, optogenetic stimuli that activated ChR2 also activated melanopsin expressed in recorded ipRGCs; consequently, we presented stimuli repeatedly to saturate the slow, melanopsin-mediated component of the response and thereby isolate any fast, ChR2-mediated component (Fig. 6B).

In $\mathrm{CRH}^{+} \mathrm{ACs}$ expressing ChR2, optogenetic stimulation generated inward currents with a mean amplitude of $-74.0 \pm 13.1 \mathrm{pA}$ and a mean latency of $1.3 \pm 0.1 \mathrm{~ms}(n=7$ cells; Fig. $6 C 1, D-F)$. At the group level, optogenetic stimulation of $\mathrm{CRH}^{+}$ACs failed to evoke significant coupling currents in M1 and M3 ipRGCs (M1: $-0.4 \pm 0.9 \mathrm{pA}, n=7, p=0.469, W=13 ; \mathrm{M} 3:-3.6 \pm 1.5 \mathrm{pA}, n=4$, $p=0.063, W=0$, one-sided Wilcoxon signed-rank tests; Fig. $6 C 2, E)$. By contrast, we observed significant inward currents in M2, M4, and M5 ipRGCs (M2: $-15.0 \pm 7.6 \mathrm{pA}$, $n=8, p=0.039, W=5$; M4: $-13.9 \pm 3.0 \mathrm{pA}, n=5, p=0.031$, $W=0$; M5: $-33.2 \pm 4.5 \mathrm{pA}, n=10, p<0.001, W=0$, onesided Wilcoxon signed-rank tests; Fig. $6 C 1, E)$, suggesting that these ipRGC types may selectively participate in electrical synapses with $\mathrm{CRH}^{+}$ACs. We further analyzed these coupling currents by defining a response threshold for each cell (i.e., $5 \times$ the SD of the baseline) and labeled each cell as responsive if it exceeded this threshold and unresponsive otherwise (Fig. 6C,D). Whereas only 3 of 8 M2 ipRGCs (mean latency $=8.6 \pm 1.1 \mathrm{~ms}$, mean amplitude $=-37.7 \pm 11.1 \mathrm{pA}$ ) and 3 of 5 M4 ipRGCs (mean latency $=15.7 \pm 2.8 \mathrm{~ms}$, mean amplitude $=-18.6 \pm 1.5 \mathrm{pA}$ ) exhibited responses under this criterion, 10 of 10 M5 ipRGCs exhibited suprathreshold responses (mean latency $=7.1 \pm 0.9 \mathrm{~ms}$; Fig. $6 E$ ). Interestingly, latencies of suprathreshold coupling currents were longer in M4, but not M2, ipRGCs than in M5 ipRGCs (M5 vs M2: $p=0.399$, $W=64.5$; M5 vs M4: $p=0.014, W=56$, Wilcoxon rank-sum tests; Fig. $6 F$ ). One possible gap junction-independent mechanism for the subset of relatively slow and/or modest coupling currents we observed could be ChR2-mediated ephaptic interactions between $\mathrm{CRH}^{+} \mathrm{AC}$ neurites and closely apposed ipRGC dendrites, as has been previously described for ChR2 in other systems (Ferenczi et al., 2016; Octeau et al., 2019). Nevertheless, these results suggest that, among ipRGC types, 

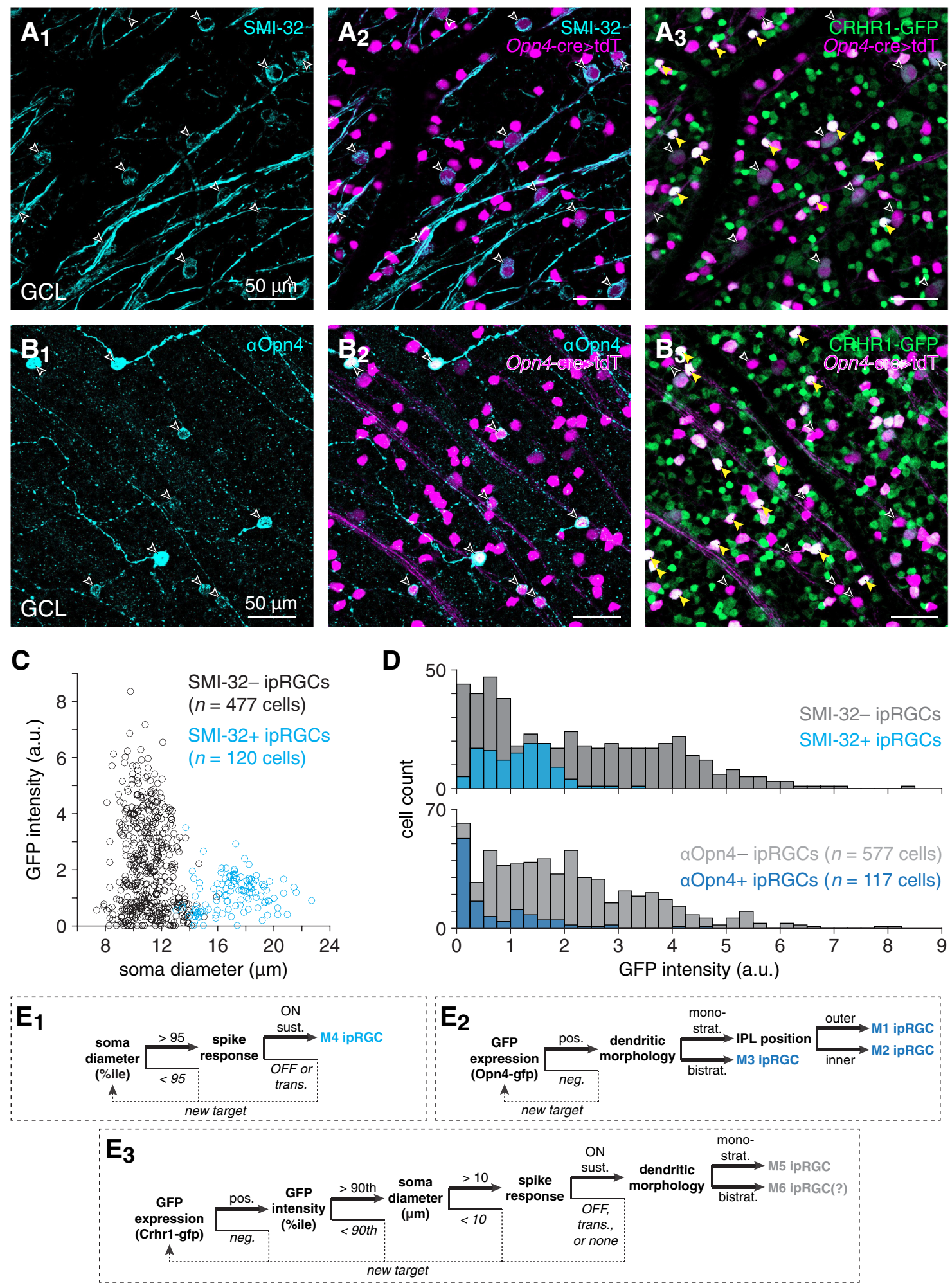

Figure 5. Identification and targeting of ipRGC types labeled in Crhr1-gfp retinas. $A$, Visualizing overlap between SMI-32- and GFP-positive cell populations in Crhr1-gfp retinas. $\boldsymbol{A}_{1}$, Confocal

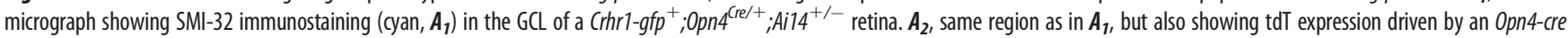
allele (Opn4-cre $>$ tdT) (magenta). Empty arrowheads indicate somas dual-labeled by SMI-32 and tdT. $\boldsymbol{A}_{3}$, same as in $\boldsymbol{A}_{2}$, but showing GFP expression (green) instead of SMI-32 staining.

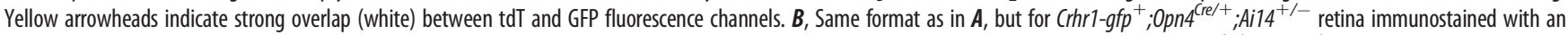

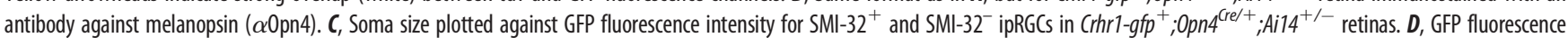

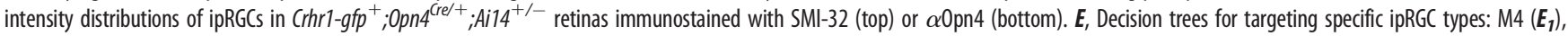
$\operatorname{M1-M3}\left(\boldsymbol{E}_{2}\right)$, and M5-M6 $\left(\boldsymbol{E}_{3}\right)$. 

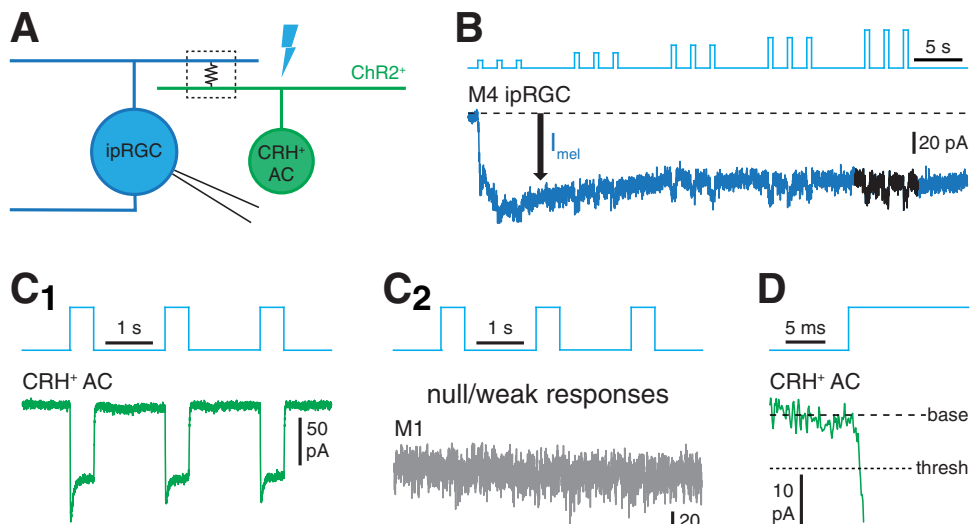

strong responses
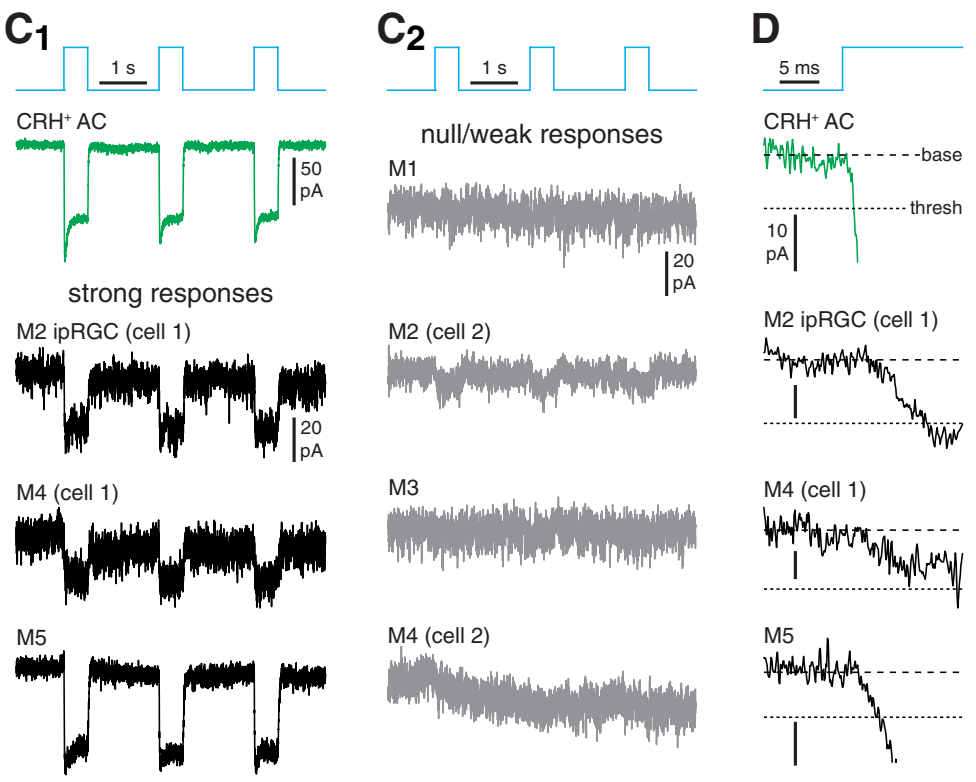

M4 (cell 1)
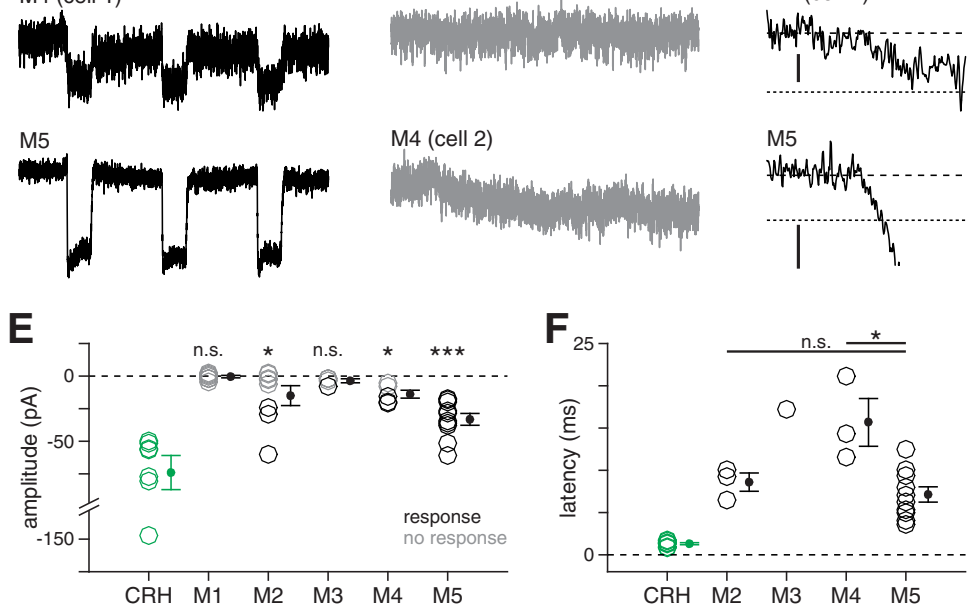

Figure 6. Selectivity of electrical coupling between ipRGC types and $\mathrm{CRH}^{+} \mathrm{ACs}$. $\boldsymbol{A}$, Circuit diagram illustrating electrophysiological assay for ipRGC-CRH ${ }^{+} \mathrm{AC}$ coupling. Optogenetic stimulation of a $\mathrm{CRH}^{+} \mathrm{AC}$ (green, right) expressing ChR2 generates a photocurrent that propagates to a recorded ipRGC (blue, left) via a gap junction-mediated electrical synapse (boxed resistor symbol). $\boldsymbol{B}$, Experimental isolation of ChR2-dependent coupling currents from melanopsin-dependent photocurrents in ipRGCs. Blue trace represents current $\left(V_{\text {hold }}=-70 \mathrm{mV}\right.$ ) recorded from an M4 ipRGC during repeated photostimulation of $\mathrm{ChR2}^{+} \mathrm{CRH}^{+} \mathrm{ACs}\left(\Phi_{\max }=4.8 \times 10^{17} \mathrm{Q} \mathrm{cm}^{-2} \mathrm{~s}^{-1}\right)$. Initially, a melanopsin-mediated inward current $\left(I_{\text {mel, }}\right.$, black arrow) dominates the measured response. During later stimuli, fast inward currents are evident. Black represents the analyzed period of the response. $\boldsymbol{C}$, Patterns of coupling currents measured in M1-M5 ipRGC types during optogenetic stimulation of $\mathrm{CRH}^{+} \mathrm{ACs} . \boldsymbol{C}_{1}$, Green trace represents lightevoked ChR2 currents recorded in a $\mathrm{CRH}^{+} \mathrm{AC}$. Black traces represent examples of fast inward currents recorded in M2, M4, and M5 ipRGCs during optogenetic stimulation of $\mathrm{CRH}^{+} \mathrm{ACs} . \mathrm{C}_{2}$, Gray traces represent examples of weak or null responses in M1-M4 ipRGCs during optogenetic stimulation of $\mathrm{CRH}^{+} \mathrm{ACs}$. D, Latencies of coupling currents in ipRGCs. Traces represent the response near stimulus onset for the cells shown in $\boldsymbol{C}_{\mathbf{7}}$. Coarse dashed lines indicate prestimulus baseline current. Fine dashed lines indicate response threshold. $\boldsymbol{E}$, ChR2 photocurrent amplitude in $\mathrm{CRH}^{+} \mathrm{ACs}(n=7$ cells) and coupling current amplitude in M1-M5 ipRGCs $(n=7, n=8, n=4, n=5$, and $n=10$ cells, respectively). $\boldsymbol{F}$, Coupling current latency for all cells in $\boldsymbol{E}$ exhibiting suprathreshold responses (CRH: $n=7 ; \mathrm{M} 2$ : $n=3$; M3: $n=1 ;$ M4: $n=3$; M5: $n=10)$. *p $<0.05$. ***p $<0.001$.

M5 ipRGCs are most strongly and reliably coupled to $\mathrm{CRH}^{+}$ ACs.

To further validate our physiological measurements of electrical coupling between $\mathrm{CRH}^{+}$ACs and specific ipRGC types, we injected individual M2, M4, and M5 ipRGCs with the gap junction-permeable tracer Neurobiotin while concurrently labeling $\mathrm{CRH}^{+} \mathrm{ACs}$ with a fluorescent reporter. For Neurobiotin-injected M2 $(n=7)$ and M4 $(n=9)$ ipRGCs, only 2 of $135(1.5 \%)$ and 0 of 95 (0\%) tracer-coupled cells, respectively, exhibited Cre- dependent fluorescence; by contrast, 25 of 60 (41.7\%) cells tracer-coupled to M5 ipRGCs $(n=5)$ exhibited Cre-dependent fluorescence (Fig. $7 A-D$ ). Indeed, at the group level, tracercoupled cells exhibiting Cre-dependent fluorescence were not distributed proportionally across the three ipRGC types $\left(p<0.001, \chi^{2}{ }_{(2)}=93.9, \chi^{2}\right.$ test; Fig. 7D). In individual ipRGCs, furthermore, the fraction of tracer-coupled cells that exhibited Cre-dependent fluorescence was significantly greater for M5 ipRGCs $(0.450 \pm 0.065)$ than for either M2 $(0.015 \pm 0.011, p=0.003$, $W=28)$ or M4 ipRGCs $(0.000 \pm 0.000, p<0.001$, $W=45$, Wilcoxon rank-sum tests; Fig. $7 E$ ). Jointly, these anatomical experiments further implicate M5 ipRGCs as the primary ipRGC population coupled to $\mathrm{CRH}^{+}$ACs.

In mice, M2 ipRGCs are electrically coupled to at least one type of monostratified, spiking, WAC (Sabbah et al., 2017). We previously reported that a morphologically and physiologically similar WAC type (CRH-3 WAC), as well as a less similar bistratified WAC type (CRH-2 WAC), exhibit Cre-dependent reporter expression in the Crh-ires-cre retina (Park et al., 2018). Both CRH-2 and CRH-3 WACs are relatively rare, and CRH-2 WACs represent only a small subset of a neuronal nitric oxide synthase-expressing $\left(\mathrm{nNOS}^{+}\right)$WAC population (Zhu et al., 2014; Park et al., 2018, 2020). CRH3 WACs were not identified in the initial morphological characterization of the Crh-ires-cre retina (Zhu et al., 2014), suggesting that they too may represent a sparsely labeled subset of another WAC population. Therefore, we hypothesized that the few M2 ipRGCs that exhibited large coupling currents (Fig. 6C1,E) or tracercoupling to putative $\mathrm{CRH}^{+}$ACs (Figs. $7 D, E$, $8 A$ ) could be explained by coupling to rare, ChR2 ${ }^{+}$CRH-3 WACs (Fig. 8B). If so, CRH-3 WACs would also exhibit rod- and cone-independent light responses generated by melanopsin activation in coupled ipRGCs. To test this hypothesis, we targeted ChR2-EYFP ${ }^{+}$CRH-3 WACs for current-clamp recording in $\mathrm{Crh}_{\mathrm{Cre}}{ }^{+/-}$; $A i 32^{+/-}$retinas. As predicted, we observed slow light responses in CRH-3 WACs during pharmacological blockade of rod- and cone-mediated input $(n=3$; Fig. $8 C)$, suggesting that these cells are coupled to at least one ipRGC type. We propose that CRH-3 WACs are coupled to M2 ipRGCs, and that expression of ChR2-EYFP in sparse CRH-3 WACs underlies occasionally large coupling currents measured in M2 ipRGCs (Fig. 6C1,E). Overall, these experiments further suggest that M2 ipRGCs provide only minor, if any, electrical synaptic ipRGC input to $\mathrm{CRH}^{+}$ACs.

Having determined that M5 ipRGCs participate in gap junction-mediated electrical synapses with $\mathrm{CRH}^{+} \mathrm{ACs}$, we next evaluated whether gap junctions mediate melanopsin-dependent light responses in $\mathrm{CRH}^{+}$ACs. Bath application of the gap junction blocker MFA $(100 \mu \mathrm{M}, \sim 15 \mathrm{~min})$ significantly reduced melanopsin-dependent light responses in $\mathrm{CRH}^{+} \mathrm{ACs}$ from $6.4 \pm 0.7 \mathrm{mV}$ 

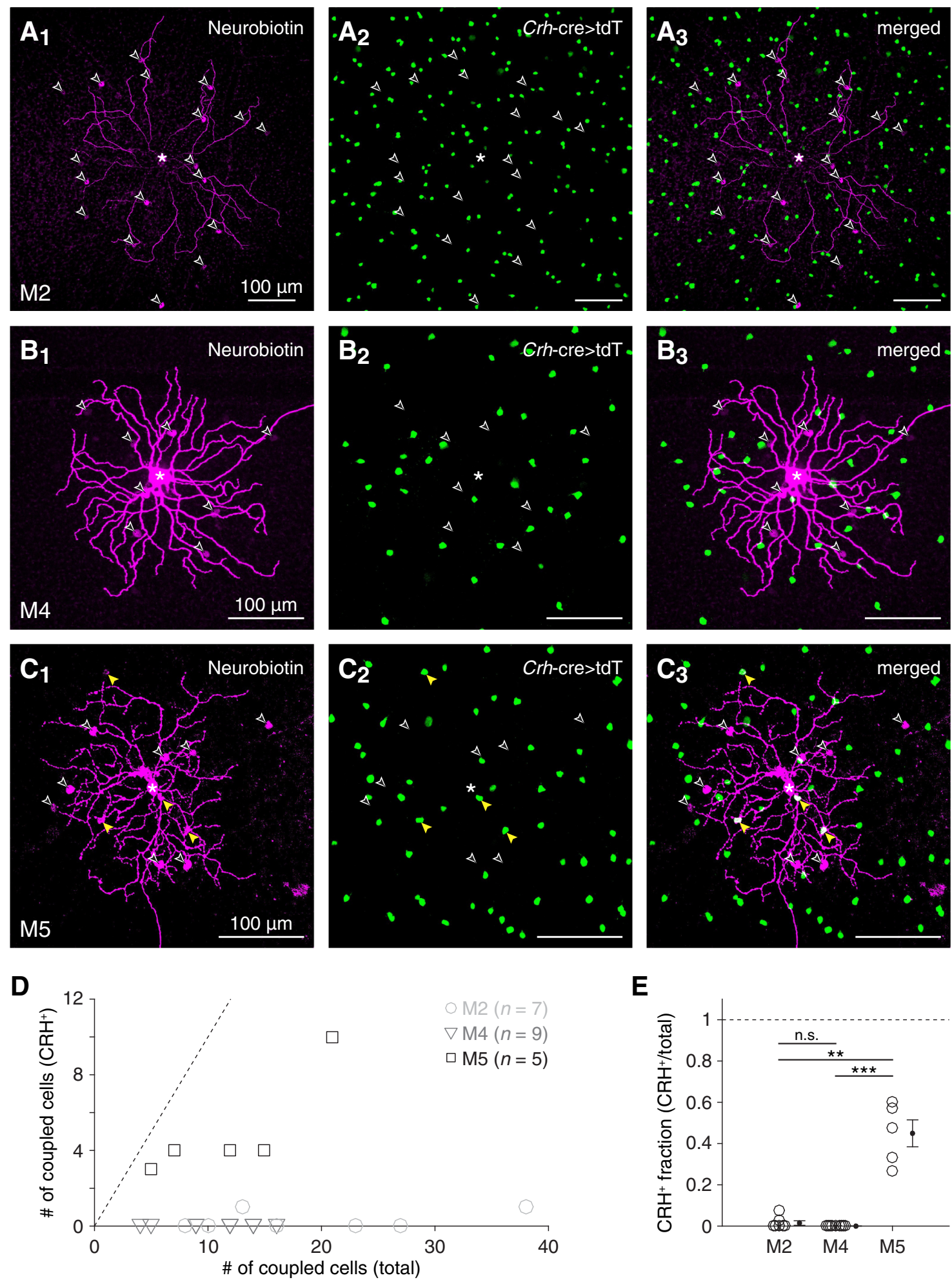

E

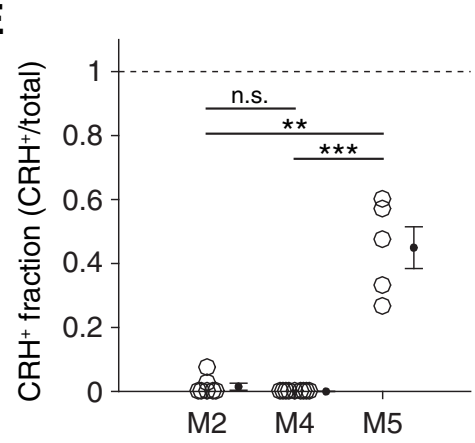

Figure 7. Selectivity of tracer coupling between ipRGC types and $\mathrm{CRH}^{+} \mathrm{ACS}$. $\boldsymbol{A}$, Visualizing overlap between cells exhibiting Crh-ires-cre-dependent fluorescence and cells tracer-coupled to an M2 ipRGC. Confocal micrographs of Neurobiotin labeling (magenta) in an injected M2 ipRGC and tracer-coupled somas $\left(\boldsymbol{A}_{1}\right)$; tdT expression (green) in putative $\mathrm{CRH}^{+} \mathrm{ACs}$, driven by a $\mathrm{Crh}^{-}$ ires-cre allele (Crh-cre $>\mathrm{tdT})\left(\boldsymbol{A}_{2}\right)$; and their overlap $\left(\boldsymbol{A}_{3}\right)$. *Soma of the Neurobiotin-injected M2 ipRGC. Empty arrowheads indicate Neurobiotin-filled somas that lack tdT expression. $\boldsymbol{B}$, Same format as in $\boldsymbol{A}$ for an M4 ipRGC. C, Same format as in $\boldsymbol{A}$ and $\boldsymbol{B}$ for an M5 ipRGC. Yellow arrowheads indicate Neurobiotin-filled somas of tdT ${ }^{+}$cells. $\boldsymbol{D}$, Total number of tracer-coupled cells plotted against number of tracer-coupled $\mathrm{CRH}^{+}$ACs for all Neurobiotin-injected M2 $(n=7), \mathrm{M} 4(n=9)$, and M5 $(n=5)$ ipRGCs. Dashed line indicates unity (i.e., cases where all tracer-coupled cells are $\left.\mathrm{CRH}^{+} \mathrm{ACs}\right)$. $\boldsymbol{E}$, Fraction of tracer-coupled cells that were putative $\mathrm{CRH}^{+} \mathrm{ACs}$, for each M2, M4, and M5 ipRGC shown in $\boldsymbol{D}$. $* * p<0.01 . * * * p<0.001$.

to $1.4 \pm 0.6 \mathrm{mV}(n=5, p=0.001, t=-8.5$, paired $t$ test; Fig. $9 A$, $B)$. By contrast, melanopsin-mediated light responses of MFAtreated M5 ipRGCs were not reduced relative to those of control M5 ipRGCs $(p=0.889, W=22$, Wilcoxon rank-sum test;
Fig. 9A,B). Additionally, we recorded melanopsin-dependent photocurrents at each of two holding potentials in individual $\mathrm{CRH}^{+}$ACs: near the reversal potential for chloride conductances $\left(\mathrm{E}_{\mathrm{Cl}} ; \mathrm{V}_{\text {hold }}=-70 \mathrm{mV}\right)$ and the reversal potential for cationic 

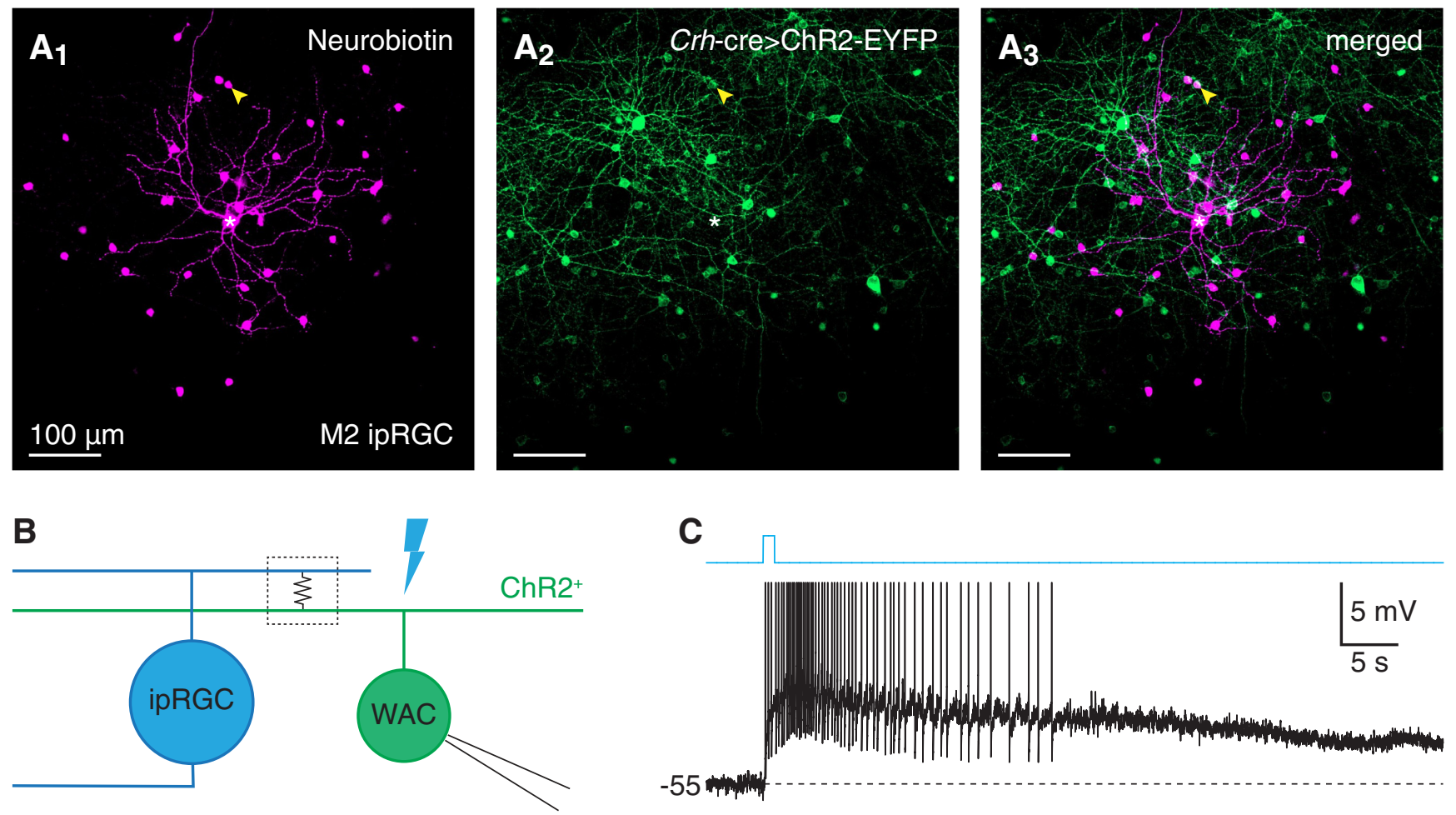

Figure 8. Rod- and cone-independent light responses of a WAC type that sparsely exhibits Crh-ires-cre-dependent fluorescence. $\boldsymbol{A}$, Tracer coupling between an M2 ipRGC and a putative $\mathrm{CRH}^{+} \mathrm{AC}$. Confocal micrographs of Neurobiotin labeling in an injected M2 ipRGC and tracer-coupled somas (Nb, magenta, $\left.\boldsymbol{A}_{7}\right)$, ChR2-EYFP expression driven by a Crh-ires-Cre allele (Crhcre $>$ ChR2-EYFP, green, $\left.A_{2}\right)$, and their overlap $\left(A_{3}\right)$ in an Opn4-gfp $; \mathrm{Crh}_{-} \mathrm{Cre}^{+/-} ; \mathrm{Aiz}^{+/-}$retina. $*$ Soma of the injected M2 ipRGC. Arrowhead indicates single Neurobiotin-filled soma exhibiting Cre-dependent fluorescence. $\boldsymbol{B}$, Circuit diagram illustrating electrical coupling between an ipRGC (left, blue) and a ChR2 ${ }^{+}$WAC (right, green). $\boldsymbol{C}$, Rod- and cone-independent voltage response of a spiking ChR2 ${ }^{+}$WAC to a $1 \mathrm{~s} \mathrm{light} \mathrm{pulse} \mathrm{in} \mathrm{a} \mathrm{Crh}-\mathrm{Cre}^{+/-} ; \mathrm{Aiz2}^{+/-}$retina $\left(\Phi_{\text {stim }}=1.5 \times 10^{16} \mathrm{Q} \mathrm{cm}^{-2} \mathrm{~s}^{-1}\right)$.

conductances $\left(\mathrm{E}_{\text {cation }} ; \mathrm{V}_{\text {hold }}=0 \mathrm{mV}\right)$. We hypothesized that, if electrical synapses mediate melanopsin-dependent light responses of $\mathrm{CRH}^{+}$ACs, melanopsin-dependent photocurrents in $\mathrm{CRH}^{+} \mathrm{ACs}$ would not be effectively nulled at $\mathrm{E}_{\text {cation }}$. Consistent with this hypothesis, mean photocurrent amplitude was not significantly reduced at $\mathrm{E}_{\text {cation }}$ compared with at $\mathrm{E}_{\mathrm{Cl}}\left(\mathrm{E}_{\mathrm{Cl}}:-10.0 \pm 1.7 \mathrm{pA} ; \mathrm{E}_{\mathrm{cation}}:-13.4 \pm 1.8 \mathrm{pA}, n=5\right.$, $p=0.945, t=-2.0$, one-sided paired $t$ test; Fig. $9 C, D)$. Together, these and all previous results suggest that electrical synapses with M5 ipRGCs mediate melanopsin-dependent light responses in $\mathrm{CRH}^{+}$ACs.

\section{Direct and indirect ipRGC targets of $\mathrm{CRH}^{+} \mathrm{AC}$-mediated synaptic inhibition}

$\mathrm{CRH}^{+}$ACs provide GABAergic inhibition to both suppressedby-contrast RGCs (Jacoby et al., 2015) and M4 ipRGCs (Park et al., 2018). Thus, through coupled $\mathrm{CRH}^{+}$ACs, M5 ipRGCs are positioned to laterally inhibit identified retinal outputs. Because the dendrites of all non-M1 ipRGC types costratify at least partially with the neurites of $\mathrm{CRH}^{+} \mathrm{ACs}$, these ipRGCs are particularly well positioned to receive synaptic inhibition from $\mathrm{CRH}^{+}$ ACs. To determine whether $\mathrm{CRH}^{+}$ACs could enable M5 ipRGCs to broadly inhibit output across ipRGC types, we optogenetically stimulated $\mathrm{CRH}^{+}$ACs while recording IPSCs in M1M5 ipRGCs (Fig. 10A). $\mathrm{CRH}^{+}$AC stimulation failed to evoke significant outward currents (i.e., putative IPSCs) in M1 and M3 ipRGCs (M1: $5.0 \pm 3.0 \mathrm{pA}, \quad n=5, \quad p=0.313, \quad W=12 ; \quad \mathrm{M} 3$ : $-3.1 \pm 3.2 \mathrm{pA}, n=5, p=0.438, W=4$, Wilcoxon signed-rank tests; Fig. $10 B$ ) but evoked significant outward currents in M2, M4, and M5 ipRGCs (M2: $29.8 \pm 3.3 \mathrm{pA}, n=11, p<0.001$,
$W=66 ; \mathrm{M} 4: 546.5 \pm 148.0 \mathrm{pA}, n=10, p=0.002, W=55 ; \quad \mathrm{M} 5$ : $49.7 \pm 13.9$ pA, $n=9, p=0.004, W=45$; Fig. $10 B, C)$.

Strikingly, only stimulus-evoked outward currents recorded in M4 and M5, but not M2, ipRGCs appeared to be associated with increased high-frequency noise, characteristic of direct synaptic transmission (Faisal et al., 2008; Ribrault et al., 2011). For each cell, we quantified this property by high-pass filtering the recording at $20 \mathrm{~Hz}$, computing the SD of periods during and before stimulation, and then computing the ratio of these two values, termed the "noise ratio" (Fig. 10D). Both M4 and M5 ipRGCs exhibited mean noise ratios significantly $>1$, indicating that outward currents evoked by optogenetic stimulation of $\mathrm{CRH}^{+} \mathrm{ACs}$ are associated with an increase in high-frequency noise in these ipRGC types (M4: 6.718 $\pm 0.466, n=10$, $p<0.001, t=12.3$; M5: 3.129 $\pm 0.327, n=9, p<0.001, t=6.5$, one-sided one-sample $t$ tests; Fig. $10 D, E)$. By contrast, outward currents recorded in M2 ipRGCs lacked such stimulus-evoked noise increases (mean noise ratio $=0.879 \pm 0.038, n=11$, $p=0.995, t=-3.2$, one-sided one-sample $t$ test; Fig. $10 D, E$ ), suggesting that these currents may not result from direct synaptic inhibition of M2 ipRGCs by $\mathrm{CRH}^{+}$ACs. In addition, for most M2 ipRGCs ( $n=7$ of 11 cells), we observed small, fast inward currents that appeared to be spikelets (Fig. 10F), indicative of electrical coupling to spiking neurons and consistent with coupling between M2 ipRGCs and spiking WACs in mice (Pérez de Sevilla Müller et al., 2010; Sabbah et al., 2017) (Fig. $8 C$ ). Jointly, these observations suggest that $\mathrm{CRH}^{+} \mathrm{ACs}$ inhibit WACs that are coupled to M2 ipRGCs, rather than inhibit M2 ipRGCs directly. Indeed, $\mathrm{CRH}^{+} \mathrm{AC}$ stimulation significantly reduced mean spikelet rates in M2 ipRGCs from $10.4 \pm 1.2 \mathrm{spi}$ kelets $s^{-1}$ to $0.6 \pm 0.4$ spikelets $s^{-1}(n=7, p<0.001, t=6.9$, 

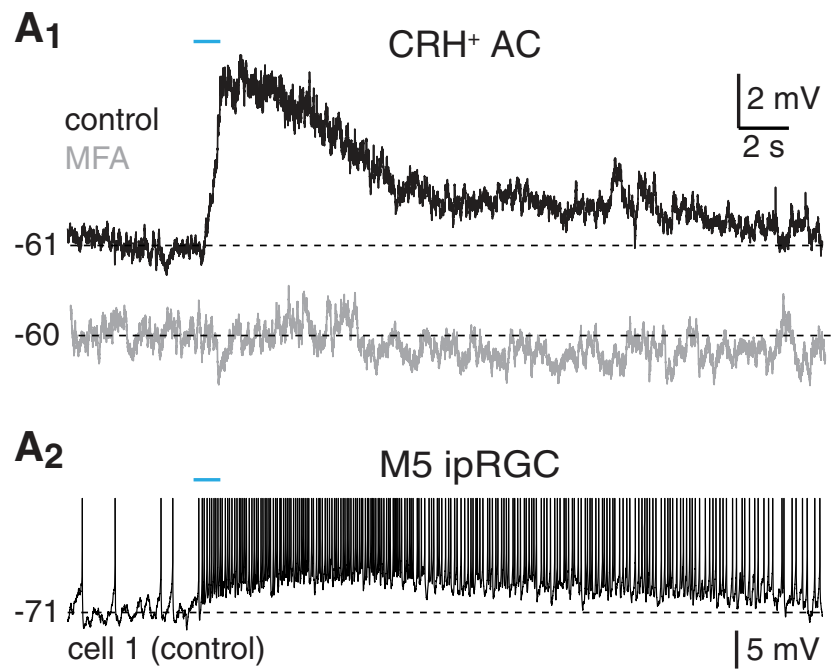

$-66$
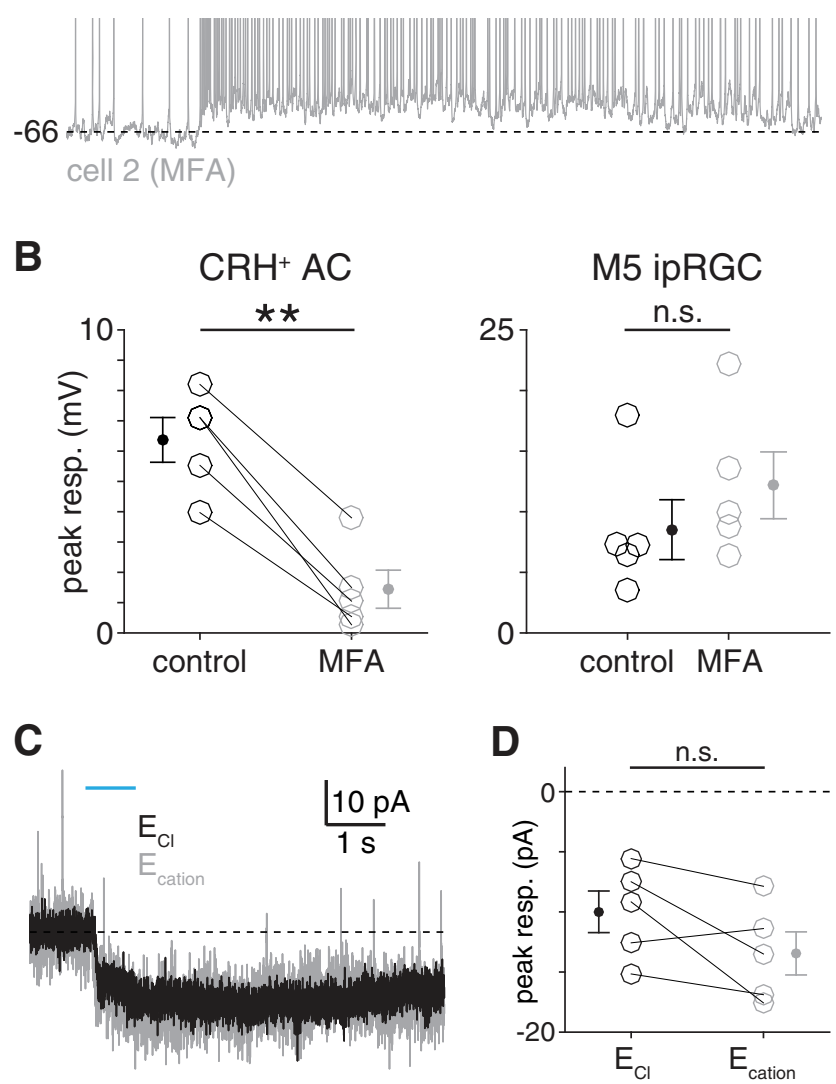

Figure 9. Gap junctions mediate melanopsin-dependent light responses of $\mathrm{CRH}^{+} \mathrm{ACs} . \boldsymbol{A}$, Melanopsin-mediated light responses of $\mathrm{CRH}^{+} \mathrm{ACs}\left(\boldsymbol{A}_{1}\right)$ and M5 ipRGCs $\left(\boldsymbol{A}_{2}\right)$ during pharmacological blockade of gap junctions. $A_{1}$, Melanopsin-dependent voltage response of a $\mathrm{CRH}^{+}$ AC before (top, black trace) and after (bottom, gray trace) bath application of MFA (100 $\mu \mathrm{m}$, $\sim 15 \mathrm{~min}$ ). $A_{2}$, Intrinsic photoresponses of a control M5 ipRGC (top, black trace) and a different M5 ipRGC exposed to MFA (bottom, gray trace). Stimulus intensity, $\Phi_{\text {stim }}=1.5 \times 10^{16}$ $Q \mathrm{~cm}^{-2} \mathrm{~s}^{-1}$. B, Peak voltage responses of CRH ${ }^{+} \mathrm{ACs}(n=5)$ before and after MFA application (left) and of M5 ipRGCs in the absence $(n=5)$ or presence $(n=5)$ of MFA (right). $\boldsymbol{C}$, Melanopsin-dependent photocurrent of $\mathrm{CRH}^{+} \mathrm{ACs}$ remains stable under changes in holding potential. Photocurrent of a $\mathrm{CRH}^{+} \mathrm{AC}$ voltage-clamped near the reversal potential for either chloride $\left(E_{c l} ; V_{\text {hold }}=-70 \mathrm{mV}\right.$, black trace) or cations $\left(E_{\text {cation; }} ; V_{\text {hold }}=0 \mathrm{mV}\right.$, gray trace). $\boldsymbol{D}$, Peak current responses in $\mathrm{CRH}^{+} \mathrm{ACs}(n=5)$ at $\mathrm{E}_{\mathrm{Cl}}$ and $\mathrm{E}_{\text {cation. }} * * p<0.01$.

paired $t$ test; Fig. 10G,H), consistent with stimulus-evoked suppression of spiking in coupled WACs and, consequently, suggesting interaction between distinct electrically coupled ipRGC-AC networks.

\section{GABAergic $\mathrm{CRH}^{+} \mathrm{AC}$ synapses support linear, low-pass transmission}

Previously, we demonstrated that $\mathrm{CRH}^{+}$synapses enable sustained GABAergic transmission onto M4 ipRGCs and pass a low-frequency $(0.5 \mathrm{~Hz})$ sinusoidal input more effectively than a higher-frequency $(5 \mathrm{~Hz})$ input (Park et al., 2018). To rigorously measure how $\mathrm{CRH}^{+} \mathrm{AC}$ synapses might transform their input signals, such as those conveyed by electrically coupled ipRGCs, over a broader range of physiologically relevant temporal frequencies, we combined continuous optogenetic stimulation with LN cascade analysis (see Materials and Methods) (Pottackal et al., 2020). Specifically, we performed optogenetic WN stimulation of $\mathrm{CRH}^{+}$ACs while recording evoked IPSCs from M4 ipRGCs and constructed LN models to quantitatively describe these IPSCs (Fig. 11A,B). For these experiments, we restricted our recordings to M4 ipRGCs because of the high signal-tonoise ratio required to generate accurate models in this paradigm. LN models generated from these IPSCs accurately predicted recorded responses to stimuli that had not been used in model construction (mean $r^{2}=0.856 \pm 0.009, n=13$; Fig. $11 B$ ). Linear filters of these LN models exhibited monophasic waveforms (Fig. 11C), characteristic of low-pass filtering. We quantified the static nonlinearity component (Fig. 11D) of each model by computing a rectification index (see Materials and Methods). Static nonlinearities obtained from IPSCs recorded in M4 ipRGCs exhibited a mean rectification index near zero $(0.079 \pm 0.017, n=13)$, indicating highly linear transmission.

Finally, we sought to identify the VGCC type that mediates GABA release from $\mathrm{CRH}^{+}$ACs onto M4 ipRGCs. In both the retina and other primary sensory circuits, synapses that support sustained synaptic transmission typically express L-type VGCCs (Pangrsic et al., 2018; Van Hook et al., 2019). We therefore hypothesized that synaptic transmission between $\mathrm{CRH}^{+} \mathrm{ACs}$ and M4 ipRGCs is likewise mediated by L-type VGCCs. Indeed, bath application of the L-type VGCC antagonist isradipine (30 $\mu \mathrm{M})$ dramatically reduced the mean amplitude of optogenetically evoked IPSCs recorded in M4 ipRGCs from $538.1 \pm 137.5 \mathrm{pA}$ to $7.1 \pm 3.1 \mathrm{pA}(n=7, p=0.008, t=3.9$, paired $t$ test; Fig. $11 E, F)$. Together, these results suggest that $\mathrm{CRH}^{+}$ACs are computationally and biophysically specialized to linearly encode sustained input signals.

\section{Discussion}

We found that $\mathrm{CRH}^{+}$ACs participate in electrical synapses with M5 ipRGCs and, hence, that light can drive $\mathrm{CRH}^{+}$ACs via at least three distinct routes: (1) direct glutamatergic input from outer photoreceptor-driven bipolar cells (BCs); (2) BC input to electrically coupled M5 ipRGCs; and (3) stimulation of melanopsin in coupled M5 ipRGCs (Fig. 12A). In turn, by modulating the membrane potential of a $\mathrm{CRH}^{+} \mathrm{AC}$, these three input sources can combine to control its transmitter outputs (i.e., GABA and $\mathrm{CRH}$ ) to specific cellular targets. Conversely, M5 ipRGC signals can propagate both (1) anterogradely to brain targets via longrange axonal projections and (2) retrogradely to intraretinal circuitry via electrical synapses with $\mathrm{CRH}^{+}$ACs. Furthermore, combined with previous studies of $\mathrm{CRH}^{+} \mathrm{AC}$ output, this study identifies three circuit motifs by which M5 ipRGCs can co-opt $\mathrm{CRH}^{+}$ACs to locally suppress retinal output (Fig. 12B1): (1) feedback inhibition (M5 ipRGCs) (Fig. 10B,C); (2) feedforward inhibition of another ipRGC type (M4 ipRGCs) (Park et al., 2018) (Fig. 10B,C); and (3) feedforward inhibition of nonipRGCs (suppressed-by-contrast RGCs) (Jacoby et al., 2015). 

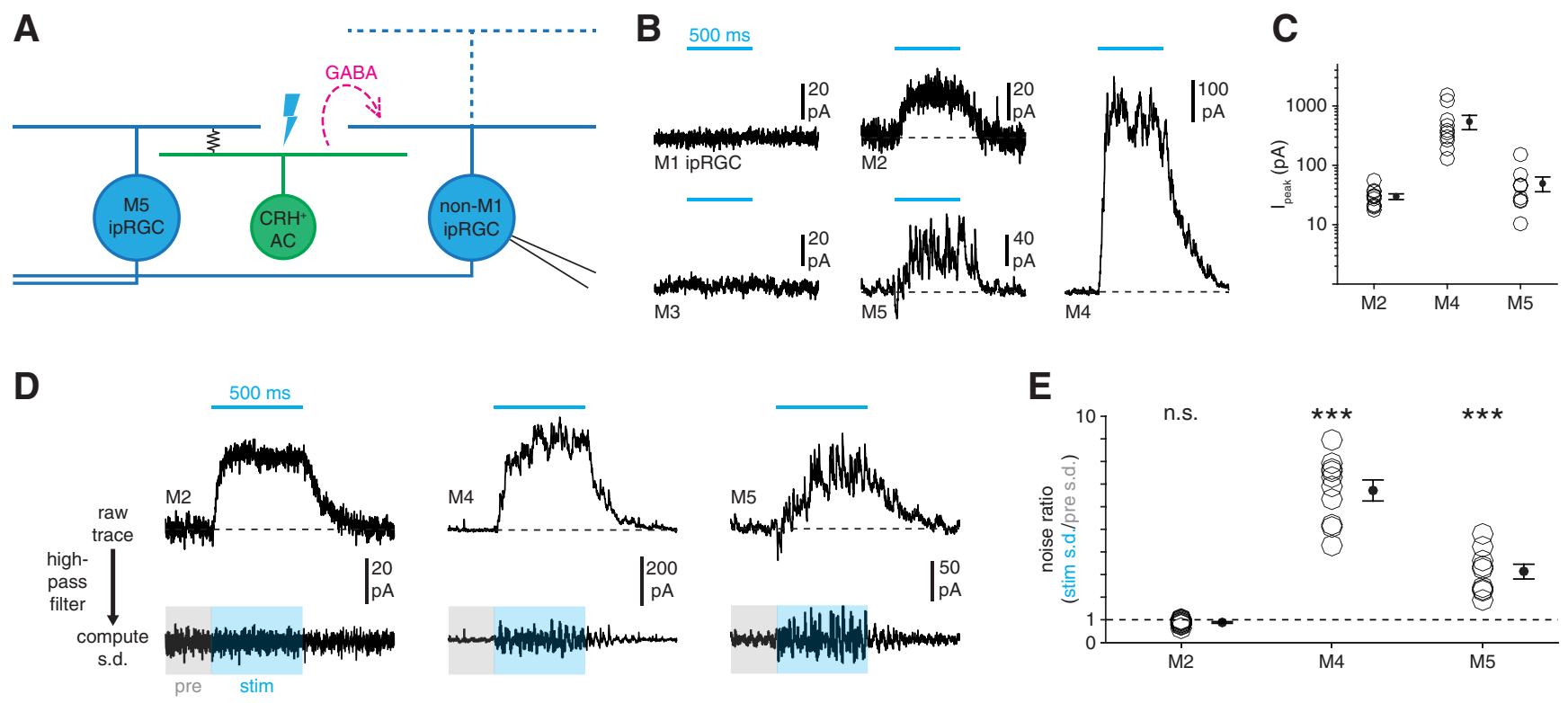

\section{E}
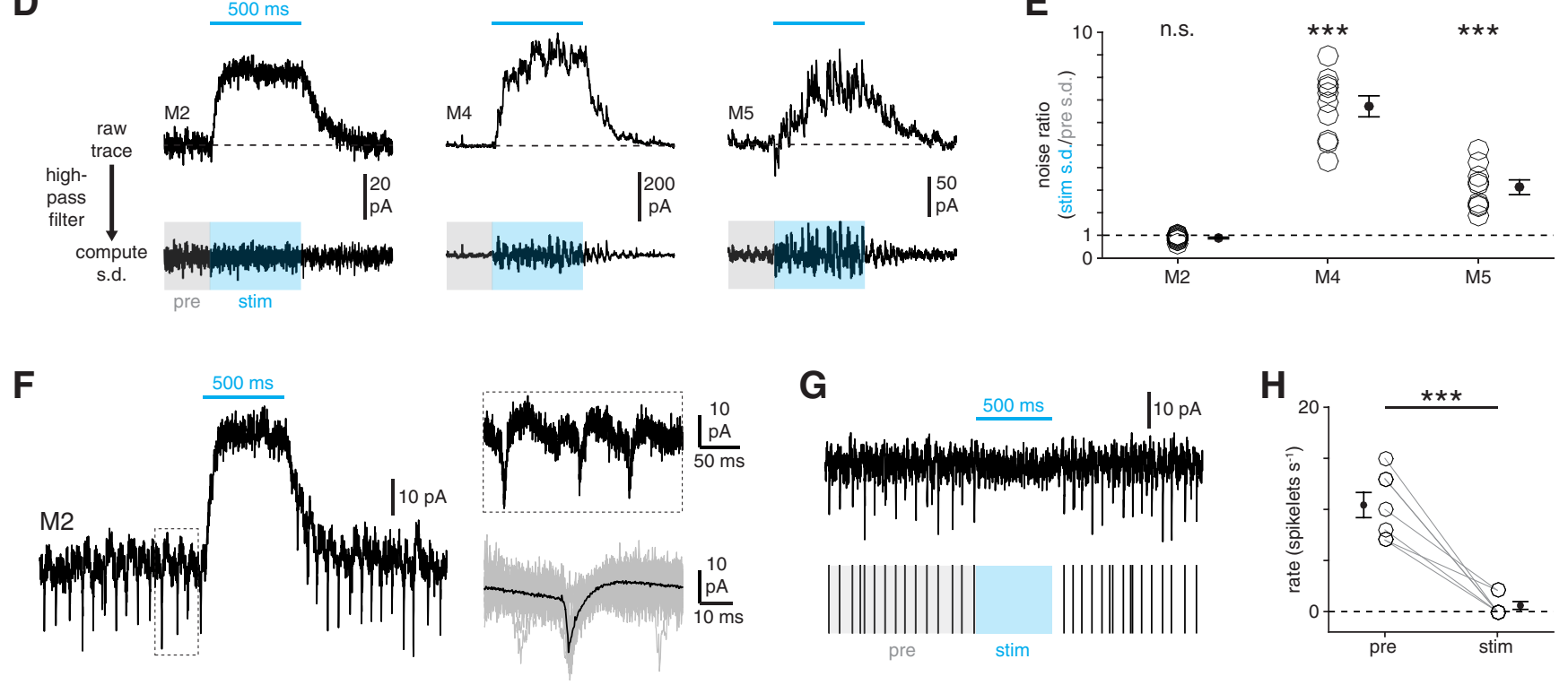

Figure 10. Cell type specificity of synaptic inhibition by $\mathrm{CRH}^{+} \mathrm{ACS}$. $A$, Circuit diagram represents GABAergic inhibition (dashed magenta arrow) of an ipRGC (right, blue) evoked by optogenetic stimulation of an M5 ipRGC-coupled CRH ${ }^{+}$AC (green, middle). $\boldsymbol{B}$, Membrane currents of M1-M5 ipRGCs during optogenetic stimulation of $\mathrm{CRH}^{+} \mathrm{ACs}_{(\mathrm{V}}\left(\mathrm{V}_{\text {hold }}=\mathrm{E}_{\text {cation }}=0 \mathrm{mV}\right)$. Cyan bars represent stimulus period $\left(\Phi_{\text {stim }}=4.8 \times 10^{17} \mathrm{Q} \mathrm{cm}^{-2} \mathrm{~s}^{-1}\right)$. C, Amplitudes of inhibitory currents evoked in M2 $(n=11), \mathrm{M} 4(n=10)$, and M5 ( $\left.n=9\right)$ ipRGCs during optogenetic stimulation of $\mathrm{CRH}^{+} \mathrm{ACS}$. D, Noise analysis of inhibitory currents evoked by $\mathrm{CRH}^{+} \mathrm{AC}$ stimulation. Top row, Currents recorded in an M2, an M4, and an M5 ipRGC during optogenetic stimulation of CRH ${ }^{+}$ ACS. Bottom row, Currents shown at top after high-pass filtering $(20 \mathrm{~Hz}$ cutoff). Noise ratio is the SD of the current during the stimulus period (stim, blue-shaded window) divided by the SD during a prestimulus period (pre, gray-shaded window). $\boldsymbol{E}$, Noise ratios in M2, M4, and M5 ipRGCs evoked by $\mathrm{CRH}^{+} \mathrm{AC}$ stimulation. $\boldsymbol{F}$, Physiological evidence for electrical coupling between M2 ipRGCs and spiking neurons. Left, Membrane current of an M2 ipRGC $\left(V_{\text {hold }}=0 \mathrm{mV}\right)$ during optogenetic stimulation of $\mathrm{CRH}^{+}$ACs. Top right, Expanded view of the boxed period at left. Bottom right, Mean (black) of 135 individual spikelets (gray, 50 shown) measured in the same cell. G, CRH ${ }^{+}$AC stimulation suppresses spikelet rate in M2 ipRGCs. Top, Black trace represents high-pass filtered membrane current of $\mathrm{M} 2$ ipRGC shown in $\boldsymbol{F}$ during $\mathrm{CRH}^{+} \mathrm{AC}$ stimulation. Bottom, Spikelet times extracted from top trace with prestimulus (pre, gray) and stimulus (stim, blue) periods indicated. $\boldsymbol{H}, \mathrm{CRH}^{+} \mathrm{AC}$ stimulation-evoked changes in spikelet rates in $\mathrm{M} 2$ ipRGCs $(n=7)$. $* * * p<0.001$.

Intriguingly, this third motif would enable ipRGCs to influence central visual circuits that lack direct axonal projections from ipRGCs.

We previously proposed that, in M4 ipRGCs, tonic inhibition from $\mathrm{CRH}^{+}$ACs serves to balance tonic excitation generated by sustained glutamate release from BCs, preventing spike response saturation and thereby extending the dynamic range of M4 ipRGCs (Park et al., 2018). However, excitatory drive to M4 ipRGCs is generated not only by BCs but also by melanopsin (Ecker et al., 2010; Estevez et al., 2012; Schmidt et al., 2014; Sonoda et al., 2018). To this point, GABAergic inhibition generated by melanopsin signaling in M5 ipRGCs could serve to balance melanopsin-mediated excitation in M4 ipRGCs. More generally, ipRGC activity drives irradiance-dependent increases in firing and information rates across a large fraction of all RGCs (Milosavljevic et al., 2018), supporting a role for ipRGC-driven inhibition in preventing saturation of RGC firing at high irradiances rather than in reducing overall firing rates. Additionally, melanopsin enhances the contrast sensitivity of M4 ipRGCs by negatively regulating a downstream potassium conductance and, consequently, increasing cellular input resistance (Sonoda et al.,
2018). Such a melanopsin-mediated increase in the excitability of an ipRGC could indirectly increase the excitability of coupled ACs, although it is currently unknown whether melanopsin functions similarly in M5 ipRGCs or any other non-M4 ipRGC type.

$\mathrm{CRH}^{+}$ACs also indirectly inhibit M2 ipRGCs, most likely through GABAergic synapses with M2 ipRGC-coupled ACs (Fig. $10 F-H)$. These ACs are predominantly spiking, polyaxonal WACs (Pérez de Sevilla Müller et al., 2010; Reifler et al., 2015; Sabbah et al., 2017). ipRGC-coupled WACs have been proposed to provide anatomical routes for the global transmission of irradiance information encoded by ipRGC activity, and in turn, to alter retinal circuit operation (Sabbah et al., 2017; Milosavljevic et al., 2018). $\mathrm{CRH}^{+}$AC-mediated inhibition of M2 ipRGCcoupled WACs could serve a role similar to that described above for M4 and M5 ipRGCs (i.e., to balance tonic excitation) to prevent spike response saturation and extend dynamic range. Although the postsynaptic targets of these M2 ipRGC-coupled WACs are currently unknown, at least one WAC type inhibits BCs that are presynaptic to M5 ipRGCs (Stabio et al., 2018). If coupled to M2 ipRGCs and postsynaptic to $\mathrm{CRH}^{+} \mathrm{ACs}$, such 
A

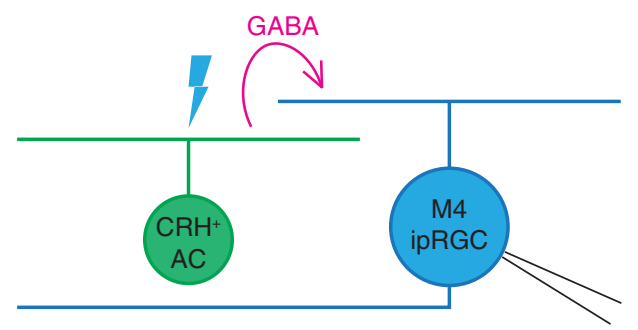

B
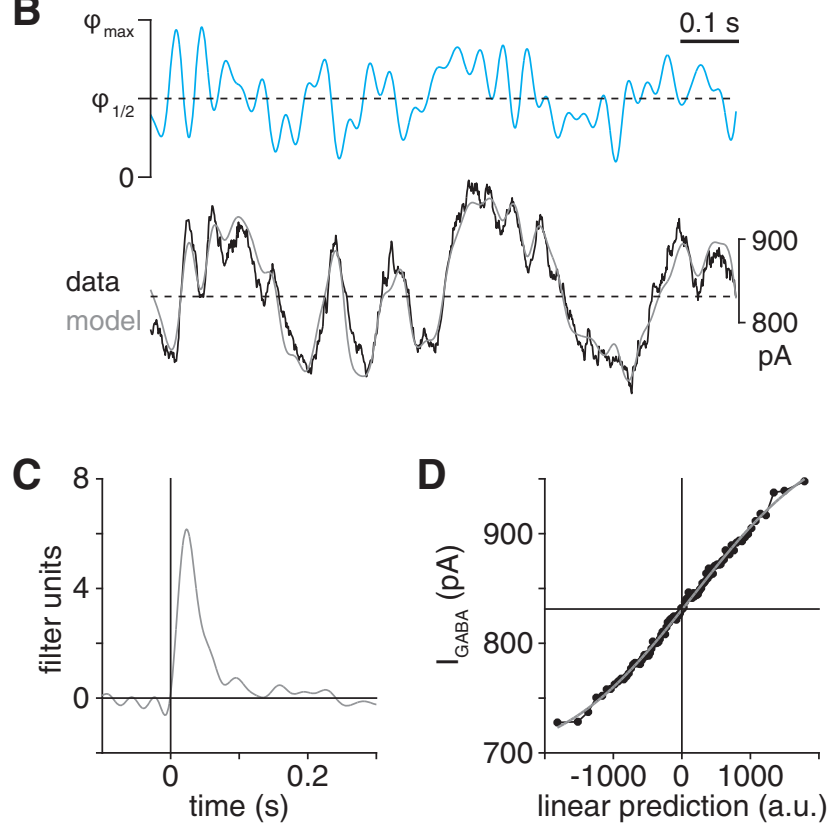

E
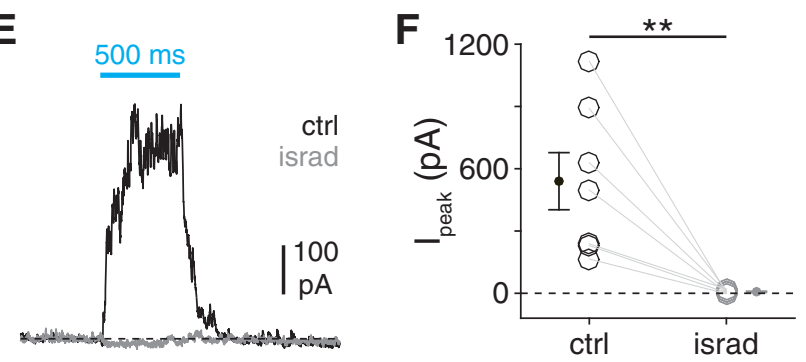

Figure 11. Computational and biophysical properties of $\mathrm{CRH}^{+} \mathrm{AC}$ synapses. $\boldsymbol{A}$, Circuit diagram illustrating GABAergic inhibition (magenta arrow) of a recorded M4 ipRGC (right, blue) evoked by optogenetic stimulation of a ChR2 ${ }^{+} \mathrm{CRH}^{+} \mathrm{AC}$ (left, green). $\boldsymbol{B}$, LN model obtained from IPSCs recorded in an M4 ipRGC during optogenetic WN stimulation of $\mathrm{CRH}^{+} \mathrm{ACs}$. Top, Cyan trace represents $1 \mathrm{~s}$ segment of an optogenetic WN stimulus $\left(\Phi_{\max }=4.8 \times 10^{17} \mathrm{Q}\right.$ $\left.\mathrm{cm}^{-2} \mathrm{~s}^{-1}\right)$. Dashed line indicates half-maximal stimulus intensity $\left(\Phi_{1 / 2}\right)$. Stimuli were designed such that average stimulus intensity was equal to $\Phi_{1 / 2}$. Bottom, Black trace represents mean IPSC obtained by averaging responses to 10 repeated trials. Gray trace represents output of LN model constructed from responses to nonrepeated stimuli. Dashed line indicates recorded response value corresponding to a linear prediction of 0 (arbitrary units, a.u.; see Materials and Methods). $\boldsymbol{C}, \boldsymbol{D}$, Linear filter ( $\boldsymbol{C}$ and static nonlinearity $(\boldsymbol{D})$ of LN model whose output is shown in $\boldsymbol{B}$. Horizontal line indicates recorded response value corresponding to a linear prediction of 0 (a.u.). Black points indicate nonlinearity computed from data. Gray curve represents fit. $\boldsymbol{E}$, IPSCS depend on L-type VGCCs. IPSCs recorded from an M4 ipRGC during optogenetic stimulation of $\mathrm{CRH}^{+} \mathrm{ACs}$ before (black) and after (gray) bath application of isradipine (israd, $30 \mu \mathrm{m})$. Cyan bar represents stimulus period $\left(\Phi_{\text {stim }}=4.8 \times 10^{17} \mathrm{Q} \mathrm{cm}^{-2}\right.$ $s^{-1}$ ). $\boldsymbol{F}$, Peak IPSC amplitudes in M4 ipRGC $(n=7$ cells) before and after isradipine application. $* * p<0.01$.
A
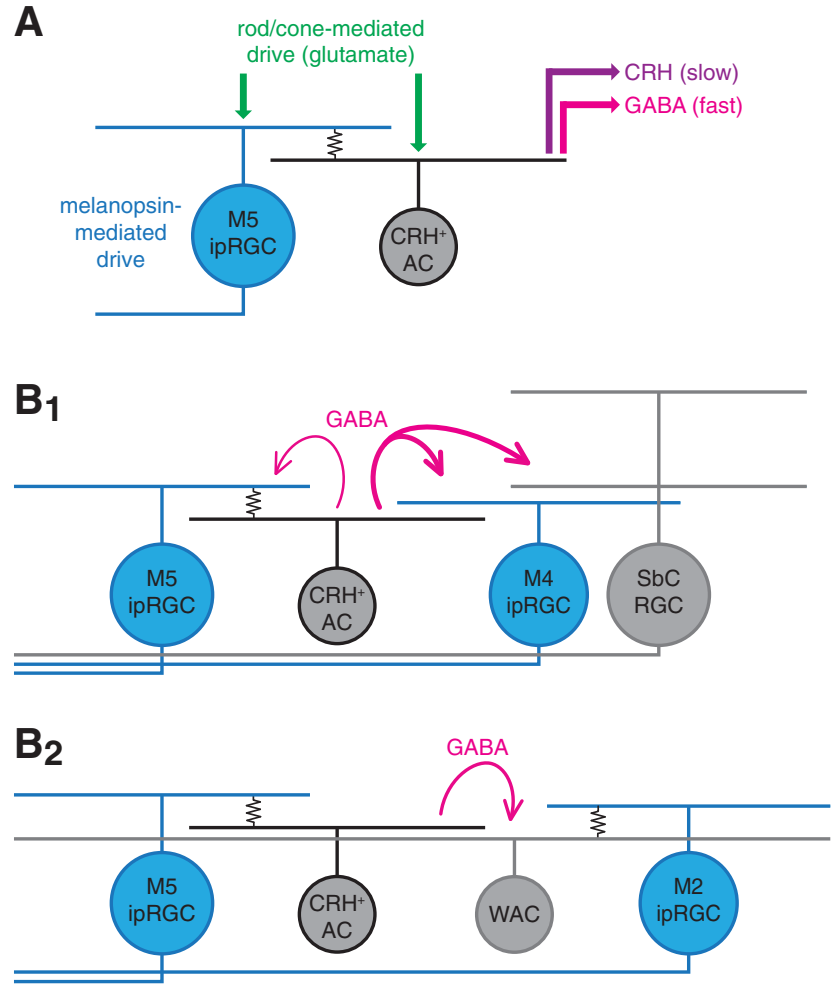

Figure 12. Proposed functions for electrical synapses between ipRGCs and $\mathrm{CRH}^{+} \mathrm{ACs} . \boldsymbol{A}$, Circuit diagram represents multiple sources of excitatory drive to an ipRGC-coupled $\mathrm{CRH}^{+} \mathrm{AC}$. $\mathrm{ACRH}^{+} \mathrm{AC}$ participates in an electrical synapse (resistor symbol) with a melanopsin-expressing M5 ipRGC. The $\mathrm{CRH}^{+} \mathrm{AC}$ directly receives excitatory glutamatergic input from BCs (green arrows), which are driven by rod and cone photoreceptor activity. Through the electrical synapse, the $\mathrm{CRH}^{+} \mathrm{AC}$ is indirectly influenced by intrinsic melanopsin- and extrinsic glutamatemediated excitatory drive of the coupled M5 ipRGC. The combined drive of these three sources modulates the release of GABA (magenta arrow) and CRH (purple arrow) onto downstream targets, potentially over different time scales. $B$, Circuit diagrams represent GABAergic signaling motifs enabled by electrical synapses between $\mathrm{CRH}^{+} \mathrm{ACs}$ and ipRGCs. $B_{1}$, GABAergic inhibition of specific ganglion cell types by a $\mathrm{CRH}^{+} \mathrm{AC}$ enables ipRGC activity to modulate retinal circuit outputs. $\mathrm{ACRH}{ }^{+} \mathrm{AC}$ (middle left, gray) provides strong feedforward GABAergic inhibition (thick magenta arrows) to an M4 ipRGC (middle right, blue) and a suppressed-by-contrast (SbC) RGC and modest feedback inhibition (fine magenta arrow) to an electrically coupled M5 ipRGC (left, blue). $\boldsymbol{B}_{\mathbf{2}}$, GABAergic inhibition of an M2 ipRGCcoupled WAC by a $\mathrm{CRH}^{+} \mathrm{AC}$ enables inhibitory interaction between distinct ipRGC-AC electrical networks. An M5 ipRGC-coupled $\mathrm{CRH}^{+} \mathrm{AC}$ (middle left, gray) provides feedforward GABAergic inhibition (magenta arrow) to an M2 ipRGC-coupled WAC (middle right, right) whose axons extend broadly throughout the retinal area.

WACs could presynaptically regulate $\mathrm{BC}$ glutamate release in an irradiance-dependent manner across the retinal area and over a wide dynamic range. Additional studies will be required to determine the genetic identities of M2 ipRGC-coupled WACs (Sabbah et al., 2017), their postsynaptic targets, their circuit functions, and the role of $\mathrm{CRH}^{+} \mathrm{AC}$-mediated inhibition in these functions.

In addition to coupling ipRGC activity to local GABAergic transmission, the circuit described above could also shape the release of $\mathrm{CRH}$, a secreted neuropeptide expressed by $\mathrm{CRH}^{+}$ ACs (Park et al., 2018). Because neuropeptide release may require prolonged presynaptic depolarization (van den Pol, 2012), the sustained visual responses of ipRGCs could be well suited for amplifying CRH release. Presently, the functions of $\mathrm{CRH}$ in the mouse retina are unknown. $\mathrm{CRH}$ immunostaining is most readily detected in $\mathrm{CRH}^{+} \mathrm{AC}$ neurites near eye opening (Park et al., 2018), potentially indicating a developmental role or, alternatively, accumulation of $\mathrm{CRH}$ before maturation of 
peptidergic release mechanisms. Although the specific retinal cell types that express CRHR1 are incompletely resolved, transgenic mice in which endogenous Crhrl promoter activity drives reporter expression exhibited labeling of the optic nerve, suggesting that CRHR1 is expressed by at least a subset of RGCs in adult mice (Kühne et al., 2012). Consistent with this result, single-cell transcriptomic analyses revealed relatively high CRHR1 mRNA levels in broad sets of RGC and AC types in adult mice (Tran et al., 2019; Yan et al., 2020). Release of CRH onto CRHR $1^{+}$RGCs by ipRGC-coupled $\mathrm{CRH}^{+}$ACs could constitute an additional signaling route by which ipRGC activity directly influences retinal output. Interestingly, M5 ipRGCs exhibit among the highest CRHR1 mRNA levels of any RGC type (Yan et al., 2020), raising the possibility that these ipRGCs drive both GABAergic and CRHergic feedback onto themselves. High CRHR1 expression in M5 ipRGCs could also explain their strong GFP labeling in Crhrl-gfp retinas, which enabled us to reliably target these cells (Figs. 4-7, 10). However, further studies will be necessary to directly validate M5 ipRGCs and other putatively CRHR1 ${ }^{+}$ RGCs as targets of CRH and, ultimately, to determine the functions of CRH release onto these cells.

Finally, although we have emphasized the potential impact of ipRGC-CRH ${ }^{+} \mathrm{AC}$ coupling on $\mathrm{CRH}^{+} \mathrm{AC}$ activity and function, these electrical synapses are not unidirectional (Fig. 6C1); therefore, the activity and function of coupled ipRGCs could likewise be affected by electrical synapses with $\mathrm{CRH}^{+}$ACs. For example, the excitatory receptive field of an individual M5 ipRGC could extend beyond that defined by its direct $\mathrm{BC}$ input as a result of glutamatergic excitation of electrically coupled $\mathrm{CRH}^{+}$ACs. In addition, if an individual $\mathrm{CRH}^{+} \mathrm{AC}$ is coupled to multiple M5 ipRGCs, it could mediate lateral spread of signals between M5 ipRGCs and, in conjunction with the nonlinear process of spike generation, enhance nonlinear spatial integration within M5 ipRGCs. Through a similar mechanism, AII ACs mediate electrical coupling within local networks of BCs, promoting lateral signal spread that combines with synaptic rectification (i.e., a nonlinear process) in $\mathrm{BC}$ axon terminals to enhance nonlinear spatial integration within BC networks (Kuo et al., 2016).

\section{References}

Allen AE, Storchi R, Martial FP, Petersen RS, Montemurro MA, Brown TM, Lucas RJ (2014) Melanopsin-driven light adaptation in mouse vision. Curr Biol 24:2481-2490.

Arroyo DA, Kirkby LA, Feller MB (2016) Retinal waves modulate an intraretinal circuit of intrinsically photosensitive retinal ganglion cells. J Neurosci 36:6892-6905.

Berson DM, Dunn FA, Takao M (2002) Phototransduction by retinal ganglion cells that set the circadian clock. Science 295:1070-1073.

Berson DM, Castrucci AM, Provencio I (2010) Morphology and mosaics of melanopsin-expressing retinal ganglion cell types in mice. J Comp Neurol 518:2405-2422.

Bleckert A, Schwartz GW, Turner MH, Rieke F, Wong RO (2014) Visual space is represented by nonmatching topographies of distinct mouse retinal ganglion cell types. Curr Biol 24:310-315.

Borghuis BG, Marvin JS, Looger LL, Demb JB (2013) Two-photon imaging of nonlinear glutamate release dynamics at bipolar cell synapses in the mouse retina. J Neurosci 33:10972-10985.

Caval-Holme F, Zhang Y, Feller MB (2019) Gap junction coupling shapes the encoding of light in the developing retina. Curr Biol 29:4024-4035.

Chen SK, Badea TC, Hattar S (2011) Photoentrainment and pupillary light reflex are mediated by distinct populations of ipRGCs. Nature 476:92-95.

Do MT (2019) Melanopsin and the intrinsically photosensitive retinal ganglion cells: biophysics to behavior. Neuron 104:205-226.

Ecker JL, Dumitrescu ON, Wong KY, Alam NM, Chen SK, LeGates T, Renna JM, Prusky GT, Berson DM, Hattar S (2010) Melanopsin-expressing retinal ganglion-cell photoreceptors: cellular diversity and role in pattern vision. Neuron 67:49-60.

Estevez ME, Fogerson PM, Ilardi MC, Borghuis BG, Chan E, Weng S, Auferkorte ON, Demb JB, Berson DM (2012) Form and function of the M4 cell, an intrinsically photosensitive retinal ganglion cell type contributing to geniculocortical vision. J Neurosci 32:13608-13620.

Faisal AA, Selen LP, Wolpert DM (2008) Noise in the nervous system. Nat Rev Neurosci 9:292-303.

Ferenczi EA, Vierock J, Atsuta-Tsunoda K, Tsunoda SP, Ramakrishnan C, Gorini C, Thompson K, Lee SY, Berndt A, Perry C, Minniberger S, Vogt A, Mattis J, Prakash R, Delp S, Deisseroth K, Hegemann P (2016) Optogenetic approaches addressing extracellular modulation of neural excitability. Sci Rep 6:23947.

Fernandez DC, Fogerson PM, Lazzerini Ospri L, Thomsen MB, Layne RM, Severin D, Zhan J, Singer JH, Kirkwood A, Zhao H, Berson DM, Hattar S (2018) Light affects mood and learning through distinct retina-brain pathways. Cell 175:71-84.e18.

Güler AD, Ecker JL, Lall GS, Haq S, Altimus CM, Liao HW, Barnard AR, Cahill H, Badea TC, Zhao H, Hankins MW, Berson DM, Lucas RJ, Yau KW, Hattar S (2008) Melanopsin cells are the principal conduits for rodcone input to non-image-forming vision. Nature 453:102-105.

Hattar S, Liao HW, Takao M, Berson DM, Yau KW (2002) Melanopsin-containing retinal ganglion cells: architecture, projections, and intrinsic photosensitivity. Science 295:1065-1070.

Hattar S, Kumar M, Park A, Tong P, Tung J, Yau KW, Berson DM (2006) Central projections of melanopsin-expressing retinal ganglion cells in the mouse. J Comp Neurol 497:326-349.

Hu EH, Pan F, Volgyi B, Bloomfield SA (2010) Light increases the gap junctional coupling of retinal ganglion cells. J Physiol 588:4145-4163.

Jacoby J, Zhu Y, DeVries SH, Schwartz GW (2015) An amacrine cell circuit for signaling steady illumination in the retina. Cell Rep 13:2663-2670.

Jarsky T, Cembrowski M, Logan SM, Kath WL, Riecke H, Demb JB, Singer JH (2011) A synaptic mechanism for retinal adaptation to luminance and contrast. J Neurosci 31:11003-11015.

Joo HR, Peterson BB, Dacey DM, Hattar S, Chen SK (2013) Recurrent axon collaterals of intrinsically photosensitive retinal ganglion cells. Vis Neurosci 30:175-182.

Justice NJ, Yuan ZF, Sawchenko PE, Vale W (2008) Type 1 corticotropinreleasing factor receptor expression reported in BAC transgenic mice: implications for reconciling ligand-receptor mismatch in the central corticotropin-releasing factor system. J Comp Neurol 511:479-496.

Kirkby LA, Feller MB (2013) Intrinsically photosensitive ganglion cells contribute to plasticity in retinal wave circuits. Proc Natl Acad Sci USA 110:12090-12095.

Krieger B, Qiao M, Rousso DL, Sanes JR, Meister M (2017) Four alpha ganglion cell types in mouse retina: function, structure, and molecular signatures. PLoS One 12:e0180091.

Kühne C, Puk O, Graw J, Hrabe de Angelis M, Schutz G, Wurst W, Deussing JM (2012) Visualizing corticotropin-releasing hormone receptor type 1 expression and neuronal connectivities in the mouse using a novel multifunctional allele. J Comp Neurol 520:3150-3180.

Kuo SP, Schwartz GW, Rieke F (2016) Nonlinear spatiotemporal integration by electrical and chemical synapses in the retina. Neuron 90:320-332.

LeGates TA, Altimus CM, Wang H, Lee HK, Yang S, Zhao H, Kirkwood A, Weber ET, Hattar S (2012) Aberrant light directly impairs mood and learning through melanopsin-expressing neurons. Nature 491:594-598.

Liao HW, Ren X, Peterson BB, Marshak DW, Yau KW, Gamlin PD, Dacey DM (2016) Melanopsin-expressing ganglion cells on macaque and human retinas form two morphologically distinct populations. J Comp Neurol 524:2845-2872.

Madisen L, Zwingman TA, Sunkin SM, Oh SW, Zariwala HA, Gu H, Ng LL, Palmiter RD, Hawrylycz MJ, Jones AR, Lein ES, Zeng H (2010) A robust and high-throughput Cre reporting and characterization system for the whole mouse brain. Nat Neurosci 13:133-140.

Madisen L, Mao T, Koch H, Zhuo JM, Berenyi A, Fujisawa S, Hsu YW, Garcia AJ, Gu X, Zanella S, Kidney J, Gu H, Mao Y, Hooks BM, Boyden ES, Buzsáki G, Ramirez JM, Jones AR, Svoboda K, Han X, et al. (2012) A toolbox of Cre-dependent optogenetic transgenic mice for light-induced activation and silencing. Nat Neurosci 15:793-802.

Milosavljevic N, Storchi R, Eleftheriou CG, Colins A, Petersen RS, Lucas RJ (2018) Photoreceptive retinal ganglion cells control the information rate of the optic nerve. Proc Natl Acad Sci USA 115:E11817-E11826. 
Nath A, Schwartz GW (2017) Electrical synapses convey orientation selectivity in the mouse retina. Nat Commun 8:2025.

Octeau JC, Gangwani MR, Allam SL, Tran D, Huang S, Hoang-Trong TM, Golshani P, Rumbell TH, Kozloski JR, Khakh BS (2019) Transient, consequential increases in extracellular potassium ions accompany channelrhodopsin2 excitation. Cell Rep 27:2249-2261.e2247.

Pangrsic T, Singer JH, Koschak A (2018) Voltage-gated calcium channels: key players in sensory coding in the retina and the inner ear. Physiol Rev 98:2063-2096.

Park SJ, Borghuis BG, Rahmani P, Zeng Q, Kim IJ, Demb JB (2015) Function and circuitry of $\mathrm{VIP}^{+}$interneurons in the mouse retina. J Neurosci 35:10685-10700.

Park SJ, Pottackal J, Ke JB, Jun NY, Rahmani P, Kim IJ, Singer JH, Demb JB (2018) Convergence and divergence of $\mathrm{CRH}$ amacrine cells in mouse retinal circuitry. J Neurosci 38:3753-3766.

Park JH, Lieberman EE, Ke JB, Rho N, Ghorbani P, Rahmani P, Jun NY, Lee HL, Kim IJ, Briggman KL, Demb JB, Singer JH (2020) Connectomic analysis reveals an interneuron with an integral role in the retinal circuit for night vision. eLife: e56077.

Pérez de Sevilla Müller L, Do MT, Yau KW, He S, Baldridge WH (2010) Tracer coupling of intrinsically photosensitive retinal ganglion cells to amacrine cells in the mouse retina. J Comp Neurol 518:4813-4824.

Pottackal J, Singer JH, Demb JB (2020) Receptoral mechanisms for fast cholinergic transmission in direction-selective retinal circuitry. Front Cell Neurosci 14:604163.

Prigge CL, Yeh PT, Liou NF, Lee CC, You SF, Liu LL, McNeill DS, Chew KS, Hattar S, Chen SK, Zhang DQ (2016) M1 ipRGCs influence visual function through retrograde signaling in the retina. J Neurosci 36:7184-7197.

Quattrochi LE, Stabio ME, Kim I, Ilardi MC, Fogerson PM, Leyrer ML, Berson DM (2019) The M6 cell: a small-field bistratified photosensitive retinal ganglion cell. J Comp Neurol 527:297-311.

Reifler AN, Chervenak AP, Dolikian ME, Benenati BA, Li BY, Wachter RD, Lynch AM, Demertzis ZD, Meyers BS, Abufarha FS, Jaeckel ER, Flannery MP, Wong KY (2015) All spiking, sustained ON displaced amacrine cells receive gap-junction input from melanopsin ganglion cells. Curr Biol 25:2763-2773.

Ribrault C, Sekimoto K, Triller A (2011) From the stochasticity of molecular processes to the variability of synaptic transmission. Nat Rev Neurosci 12:375-387.

Rodriguez AR, de Sevilla Müller LP, Brecha NC (2014) The RNA binding protein RBPMS is a selective marker of ganglion cells in the mammalian retina. J Comp Neurol 522:1411-1443.

Sabbah S, Berg D, Papendorp C, Briggman KL, Berson DM (2017) A Cre mouse line for probing irradiance- and direction-encoding retinal networks. eNeuro 4:ENEURO.0065-17.2017.

Schmidt TM, Kofuji P (2009) Functional and morphological differences among intrinsically photosensitive retinal ganglion cells. J Neurosci 29:476-482.

Schmidt TM, Kofuji P (2011) Structure and function of bistratified intrinsically photosensitive retinal ganglion cells in the mouse. J Comp Neurol 519:1492-1504

Schmidt TM, Chen SK, Hattar S (2011) Intrinsically photosensitive retinal ganglion cells: many subtypes, diverse functions. Trends Neurosci 34:572-580.

Schmidt TM, Alam NM, Chen S, Kofuji P, Li W, Prusky GT, Hattar S (2014) A role for melanopsin in alpha retinal ganglion cells and contrast detection. Neuron 82:781-788.
Schubert T, Degen J, Willecke K, Hormuzdi SG, Monyer H, Weiler R (2005) Connexin 36 mediates gap junctional coupling of alpha-ganglion cells in mouse retina. J Comp Neurol 485:191-201.

Sekaran S, Lupi D, Jones SL, Sheely CJ, Hattar S, Yau KW, Lucas RJ, Foster RG, Hankins MW (2005) Melanopsin-dependent photoreception provides earliest light detection in the mammalian retina. Curr Biol 15:10991107.

Sonoda T, Lee SK, Birnbaumer L, Schmidt TM (2018) Melanopsin phototransduction is repurposed by ipRGC subtypes to shape the function of distinct visual circuits. Neuron 99:754-767.e754.

Sonoda T, Okabe Y, Schmidt TM (2020) Overlapping morphological and functional properties between M4 and M5 intrinsically photosensitive retinal ganglion cells. J Comp Neurol 528:1028-1040.

Stabio ME, Sabbah S, Quattrochi LE, Ilardi MC, Fogerson PM, Leyrer ML, Kim MT, Kim I, Schiel M, Renna JM, Briggman KL, Berson DM (2018) The M5 cell: a color-opponent intrinsically photosensitive retinal ganglion cell. Neuron 97:150-163.e154.

Taniguchi H, He M, Wu P, Kim S, Paik R, Sugino K, Kvitsiani D, Kvitsani D, Fu Y, Lu J, Lin Y, Miyoshi G, Shima Y, Fishell G, Nelson SB, Huang ZJ (2011) A resource of Cre driver lines for genetic targeting of GABAergic neurons in cerebral cortex. Neuron 71:995-1013.

Tikidji-Hamburyan A, Reinhard K, Seitter H, Hovhannisyan A, Procyk CA, Allen AE, Schenk M, Lucas RJ, Münch TA (2015) Retinal output changes qualitatively with every change in ambient illuminance. Nat Neurosci 18:66-74.

Tran NM, Shekhar K, Whitney IE, Jacobi A, Benhar I, Hong G, Yan W, Adiconis X, Arnold ME, Lee JM, Levin JZ, Lin D, Wang C, Lieber CM, Regev A, He Z, Sanes JR (2019) Single-cell profiles of retinal ganglion cells differing in resilience to injury reveal neuroprotective genes. Neuron 104:1039-1055.e1012.

Tu DC, Zhang D, Demas J, Slutsky EB, Provencio I, Holy TE, Van Gelder RN (2005) Physiologic diversity and development of intrinsically photosensitive retinal ganglion cells. Neuron 48:987-999.

van den Pol AN (2012) Neuropeptide transmission in brain circuits. Neuron 76:98-115.

Van Hook MJ, Nawy S, Thoreson WB (2019) Voltage- and calcium-gated ion channels of neurons in the vertebrate retina. Prog Retin Eye Res 72:100760.

Viney TJ, Balint K, Hillier D, Siegert S, Boldogkoi Z, Enquist LW, Meister M, Cepko CL, Roska B (2007) Local retinal circuits of melanopsin-containing ganglion cells identified by transsynaptic viral tracing. Curr Biol 17:981-988.

Völgyi B, Abrams J, Paul DL, Bloomfield SA (2005) Morphology and tracer coupling pattern of alpha ganglion cells in the mouse retina. J Comp Neurol 492:66-77.

Yan W, Laboulaye MA, Tran NM, Whitney IE, Benhar I, Sanes JR (2020) Molecular identification of sixty-three amacrine cell types completes a mouse retinal cell atlas. J Neurosci 40:5177-5195.

Zhang DQ, Wong KY, Sollars PJ, Berson DM, Pickard GE, McMahon DG (2008) Intraretinal signaling by ganglion cell photoreceptors to dopaminergic amacrine neurons. Proc Natl Acad Sci USA 105:14181-14186.

Zhao X, Stafford BK, Godin AL, King WM, Wong KY (2014) Photoresponse diversity among the five types of intrinsically photosensitive retinal ganglion cells. J Physiol 592:1619-1636.

Zhu Y, Xu J, Hauswirth WW, DeVries SH (2014) Genetically targeted binary labeling of retinal neurons. J Neurosci 34:7845-7861. 